\title{
Total Synthesis of the Proposed Structure of
}

\section{Uncarialin A}

Niklāvs Ūdris, Kristaps Jaudzems and Gints Smits*

\section{Corresponding Author}

Gints Smits - Latvian Institute of Organic Synthesis, Aizkraukles 21, Riga, Latvia, LV1006; Email: gintssmits@,osi.lv

https://orcid.org/0000-0001-5044-4169

\section{Table of content}

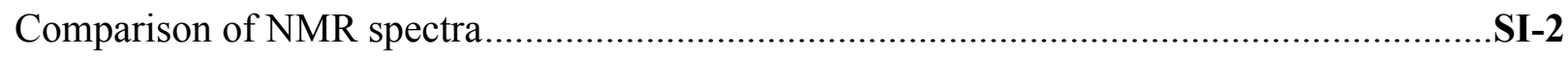

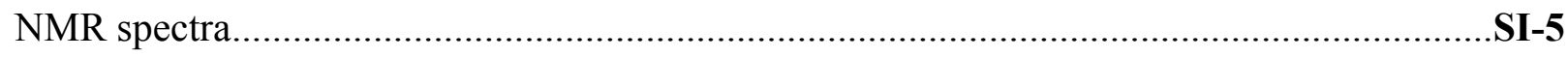




\section{Comparison of NMR data}

Uncarialin A (1)

Table S1. ${ }^{1} \mathrm{H}$ Data comparison of the synthetic $\boldsymbol{E}-\mathbf{1}$ and the literature

\begin{tabular}{|l|l|l|l|}
\hline no. & $\begin{array}{l}\text { Literature, }{ }^{1} \text { 600 MHz, } \\
\text { Methanol- } d 4, \delta \mathrm{H}(\mathrm{J} \text { in Hz) }\end{array}$ & $\begin{array}{l}\text { Synthetic, free base, } 400 \mathrm{MHz}, \\
\text { Methanol- } d 4, \delta \mathrm{H}(\mathrm{J} \text { in Hz) }\end{array}$ & $\begin{array}{l}\text { Synthetic, TFA salt, 400 MHz, } \\
\text { Methanol- } d 4, \delta \mathrm{H}(\mathrm{J} \text { in Hz) }\end{array}$ \\
\hline 3 & $5.05, \mathrm{~s}$ & $3.15, \mathrm{~d}(11.2)$ & $4.43, \mathrm{~d}(12.2)$ \\
\hline 5 & $\begin{array}{l}3.71, \mathrm{dd}(12.6,6.2) \\
3.58, \mathrm{td}(12.6,5.4)\end{array}$ & $\begin{array}{l}2.52, \mathrm{~m} \\
2.99, \mathrm{~m}\end{array}$ & $\begin{array}{l}3.44, \mathrm{td}(12.3,5.4) \\
3.79, \mathrm{dd}(12.3,6.0)\end{array}$ \\
\hline 6 & $3.02, \mathrm{dd}(16.8,5.4)$ & $2.66 \mathrm{dd}(14.1,4.9)$ & $3.02, \mathrm{dd}(16.2,5.3)$ \\
& $3.19, \mathrm{dd}(16.8,6.2)$ & $2.99, \mathrm{~m}$ & $3.29, \mathrm{~m}$ \\
\hline 9 & $7.49, \mathrm{~d}(7.8)$ & $7.37, \mathrm{~d}(7.3)$ & $7.48, \mathrm{~d}(7.9)$ \\
\hline 10 & $7.08, \mathrm{t}(7.5)$ & $6.96, \mathrm{~d}(7.1)$ & $7.08, \mathrm{dd}(8.0,7.1)$ \\
\hline 11 & $7.17, \mathrm{t}(7.5)$ & $7.02, \mathrm{~d}(7.0)$ & $7.17, \mathrm{dd}(8.2,7.1)$ \\
\hline 12 & $7.40, \mathrm{~d}(8.0)$ & $7.28, \mathrm{~d}(8.0)$ & $7.38, \mathrm{~d}(8.1)$ \\
\hline 14 & $2.77, \mathrm{t}(11.2)$ & $2.08, \mathrm{~d}(13.2)$ & $2.50, \mathrm{~d}(14.4)$ \\
& $2.44, \mathrm{~m}$ & $2.48, \mathrm{~m}$ & $2.76, \mathrm{dt}(14.8,12.7)$ \\
\hline 15 & $2.48, \mathrm{~m}$ & $3.08, \mathrm{~m}$ & $3.37, \mathrm{~m}$ \\
\hline 17 & $7.52, \mathrm{~s}$ & $7.53, \mathrm{~s}$ & $7.62, \mathrm{~s}$ \\
\hline 18 & $0.77, \mathrm{t}(7.4)$ & $0.88, \mathrm{t}(7.4)$ & $0.95, \mathrm{t}(7.4)$ \\
\hline 19 & $1.39, \mathrm{~m}$ & $1.28, \mathrm{~m}$ & $1.47, \mathrm{~m}$ \\
& $0.93, \mathrm{~m}$ & $1.77, \mathrm{~m}$ & $1.67, \mathrm{ddq}(14.4,11.7,7.3)$ \\
\hline 20 & $2.44, \mathrm{~m}$ & $1.65, \mathrm{~m}$ & $3.02, \mathrm{~m}$ \\
\hline 21 & $3.41, \mathrm{dd}(12.1,2.9)$ & $2.44, \mathrm{~m}$ & $3.85, \mathrm{~s}$ \\
\hline 17 & $2.92, \mathrm{dd}(12.1,10.5)$ & $3.07, \mathrm{~m}$ & $3.72, \mathrm{~s}$ \\
\hline 22 & $3.81, \mathrm{~s}$ & $3.77, \mathrm{~s}$ & \\
\hline
\end{tabular}

1. Journal of Natural Products, 2019, 82 (12), 3302-3310. 
Table S2. ${ }^{13} \mathrm{C}$ Data comparison of the synthetic $\boldsymbol{E}-\mathbf{1}$ and the literature

\begin{tabular}{|c|c|c|c|}
\hline no. & $\begin{array}{l}\text { Literature, }^{1} \\
150 \mathrm{MHz}, \text { Methanol- } d 4\end{array}$ & $\begin{array}{l}\text { Synthetic, free base, } \\
100 \mathrm{MHz}, \text { Methanol-d4 }\end{array}$ & $\begin{array}{l}\text { Synthetic, TFA salt, } \\
100 \mathrm{MHz}, \text { Methanol- } d 4\end{array}$ \\
\hline 2 & 127.6 & 136.3 & 129.4 \\
\hline 3 & 57.4 & 63.6 & 64.5 \\
\hline 5 & 52.4 & 54.9 & 54.8 \\
\hline 6 & 17.0 & 22.4 & 19.9 \\
\hline 7 & 106.4 & 107.7 & 106.8 \\
\hline 8 & 127.6 & 128.5 & 127.4 \\
\hline 9 & 119.4 & 118.5 & 119.2 \\
\hline 10 & 120.9 & 119.6 & 120.8 \\
\hline 11 & 123.6 & 121.7 & 123.7 \\
\hline 12 & 112.7 & 111.9 & 112.6 \\
\hline 13 & 138.6 & 138.0 & 138.5 \\
\hline 14 & 30.8 & 30.5 & 28.5 \\
\hline 15 & 33.5 & 41.2 & 38.8 \\
\hline 16 & 110.3 & 112.3 & 110.0 \\
\hline 17 & 163.0 & 162.4 & 163.3 \\
\hline 18 & 10.9 & 13.3 & 12.6 \\
\hline 19 & 24.7 & 20.3 & 19.4 \\
\hline 20 & 38.3 & 42.1 & 40.4 \\
\hline 21 & 51.2 & 58.9 & 57.0 \\
\hline 22 & 169.5 & 170.8 & 170.0 \\
\hline 17-OMe & 62.6 & 62.2 & 62.6 \\
\hline $22-\mathrm{OMe}$ & 51.9 & 51.8 & 52.0 \\
\hline
\end{tabular}




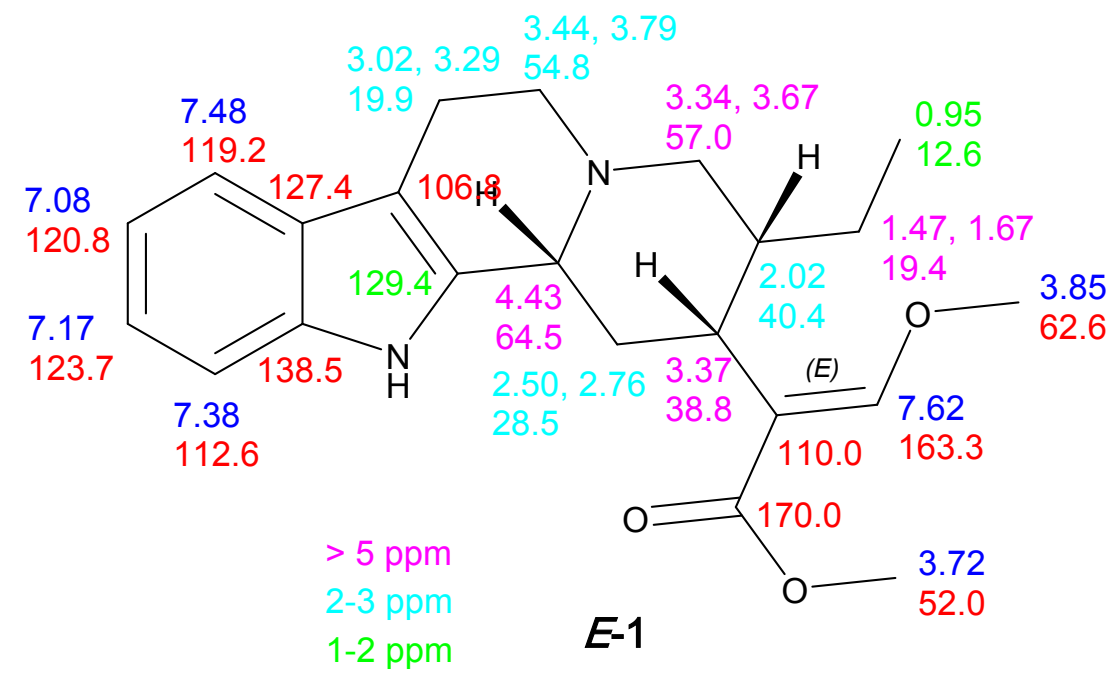

Figure S1. ${ }^{13} \mathrm{C}$ chemical shift differences between $\boldsymbol{E}$-1 TFA salt and the literature values

Table S3. ${ }^{1} \mathrm{H}$ Data comparison of the synthetic $\boldsymbol{E}-\mathbf{1}$ and (-) corynantheidine (2)

\begin{tabular}{|l|l|}
\hline Literature, ${ }^{2} 400 \mathrm{MHz}, \mathrm{CDCl}_{3}$ & Synthetic, $400 \mathrm{MHz}, \mathrm{CDCl}_{3}$ \\
\hline $0.87(\mathrm{t}, \mathrm{J}=7.5 \mathrm{~Hz}, 3 \mathrm{H})$ & $0.88(\mathrm{t}, \mathrm{J}=7.4 \mathrm{~Hz}, 3 \mathrm{H})$ \\
\hline $1.85-1.60(\mathrm{~m}, 4 \mathrm{H})$ & $1.88-1.59(\mathrm{~m}, 4 \mathrm{H})$ \\
\hline $2.71-2.48(\mathrm{~m}, 4 \mathrm{H})$ & $2.75-2.45(\mathrm{~m}, 4 \mathrm{H})$ \\
\hline $3.08-2.95(\mathrm{~m}, 4 \mathrm{H})$ & $3.10-2.91(\mathrm{~m}, 4 \mathrm{H})$ \\
\hline $3.21-3.19(\mathrm{~m}, 1 \mathrm{H})$ & $3.24-3.14(\mathrm{~m}, 1 \mathrm{H})$ \\
\hline $3.71(\mathrm{~s}, 3 \mathrm{H})$ & $3.72(\mathrm{~s}, 3 \mathrm{H})$ \\
\hline $3.73(\mathrm{~s}, 3 \mathrm{H})$ & $3.73(\mathrm{~s}, 3 \mathrm{H})$ \\
\hline $7.13-7.06(\mathrm{~m}, 2 \mathrm{H})$ & $7.16-7.03(\mathrm{~m}, 2 \mathrm{H})$ \\
\hline $7.30(\mathrm{~d}, \mathrm{~J}=8.0 \mathrm{~Hz}, 1 \mathrm{H})$ & $7.29(\mathrm{~d}, \mathrm{~J}=7.3 \mathrm{~Hz}, 1 \mathrm{H})$ \\
\hline $7.44(\mathrm{~s}, 1 \mathrm{H})$ & $7.44(\mathrm{~s}, 1 \mathrm{H})$ \\
\hline $7.46(\mathrm{~d}, \mathrm{~J}=7.5 \mathrm{~Hz}, 1 \mathrm{H})$ & $7.47(\mathrm{~d}, \mathrm{~J}=7.3 \mathrm{~Hz}, 1 \mathrm{H})$ \\
\hline $7.72(\mathrm{brs}, 1 \mathrm{H})$ & - \\
\hline
\end{tabular}

Table S4. ${ }^{13} \mathrm{C}$ Data comparison of the synthetic $\boldsymbol{E}-1$ and (-) corynantheidine (2)

\begin{tabular}{|l|l|}
\hline Literature, ${ }^{2} 125 \mathrm{MHz}, \mathrm{CDCl}_{3}$ & Synthetic, $100 \mathrm{MHz}, \mathrm{CDCl}_{3}$ \\
\hline 12.9 & 13.0 \\
\hline 19.1 & 19.2 \\
\hline 21.9 & 22.0 \\
\hline
\end{tabular}

${ }^{2}$ Eur. J. Org. Chem. 2011, 14, 2606-2609. 


\begin{tabular}{|l|l|}
\hline 29.9 & 30.0 \\
\hline 39.9 & 40.1 \\
\hline 40.7 & 40.8 \\
\hline 51.4 & 51.5 \\
\hline 53.5 & 53.6 \\
\hline 57.8 & 57.9 \\
\hline 61.2 & 61.4 \\
\hline 61.5 & 61.7 \\
\hline 108.1 & 108.1 \\
\hline 110.5 & 110.7 \\
\hline 111.5 & 111.6 \\
\hline 118.0 & 118.2 \\
\hline 119.3 & 119.4 \\
\hline 121.1 & 121.2 \\
\hline 127.5 & 127.6 \\
\hline 135.6 & 135.6 \\
\hline 135.9 & 135.9 \\
\hline 160.5 & 160.7 \\
\hline 169.2 & 169.4 \\
\hline
\end{tabular}


NMR Spectra

CASL2-GS-354.10.1.1r

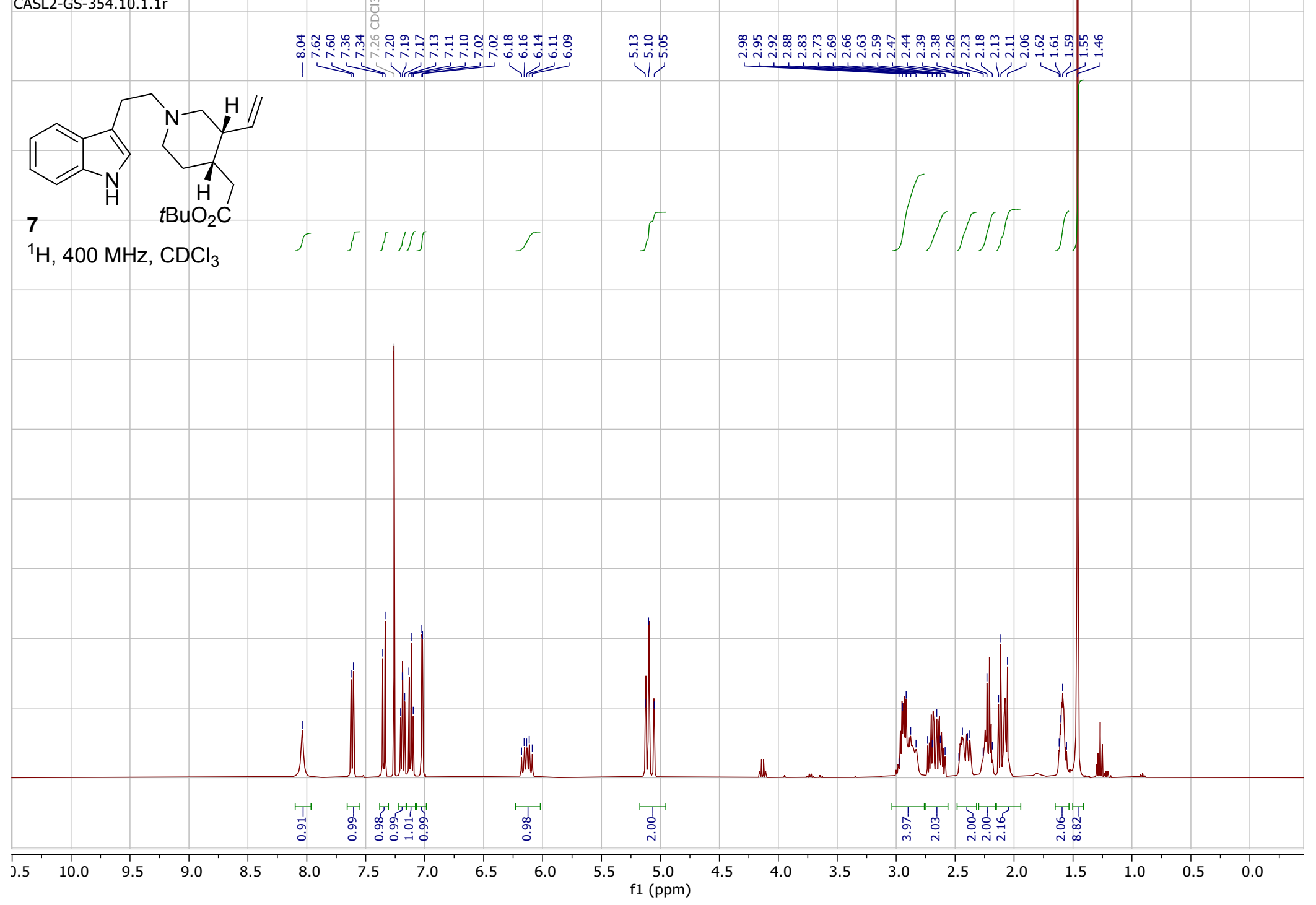

SI-6 

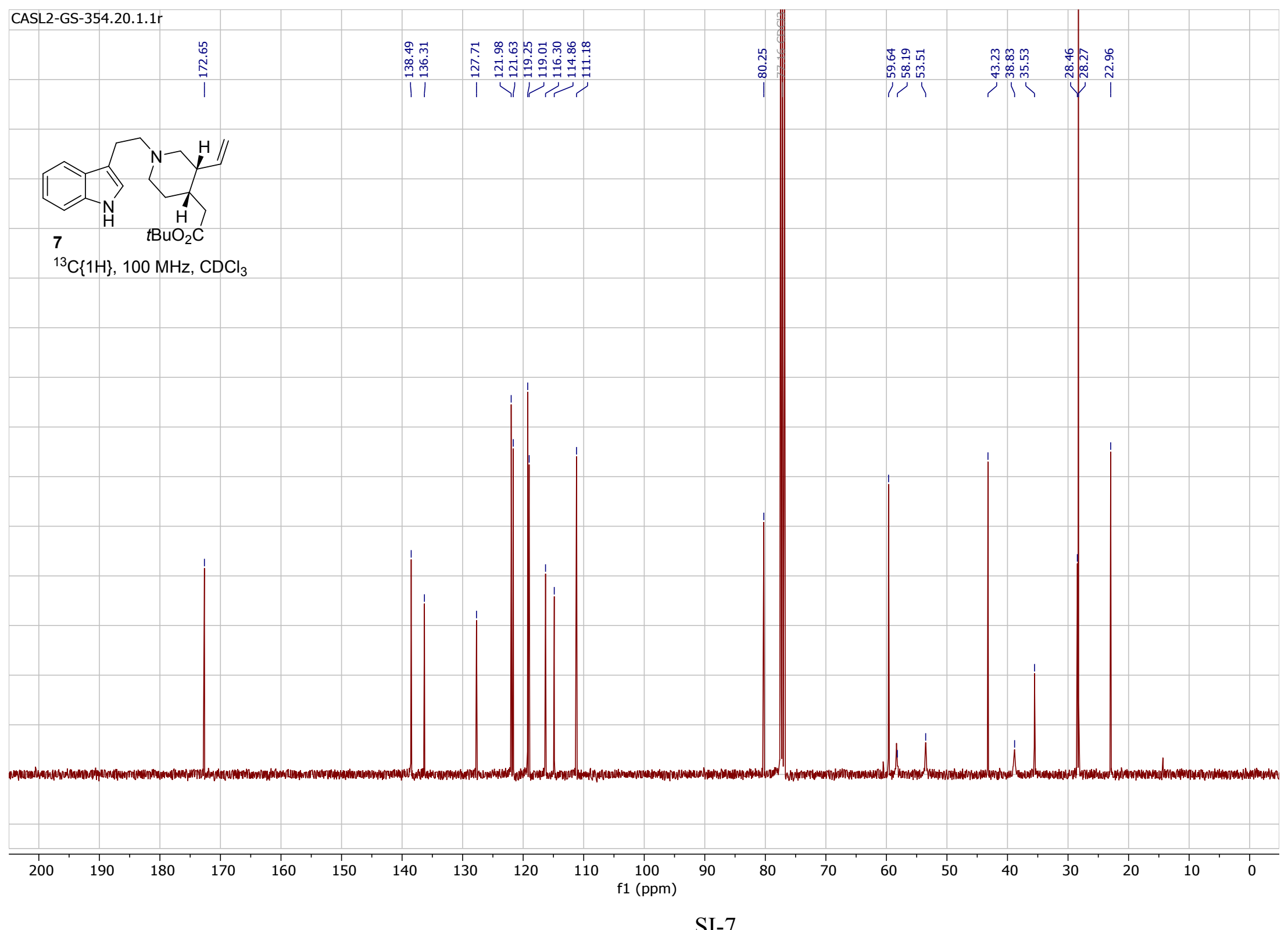

SI-7 


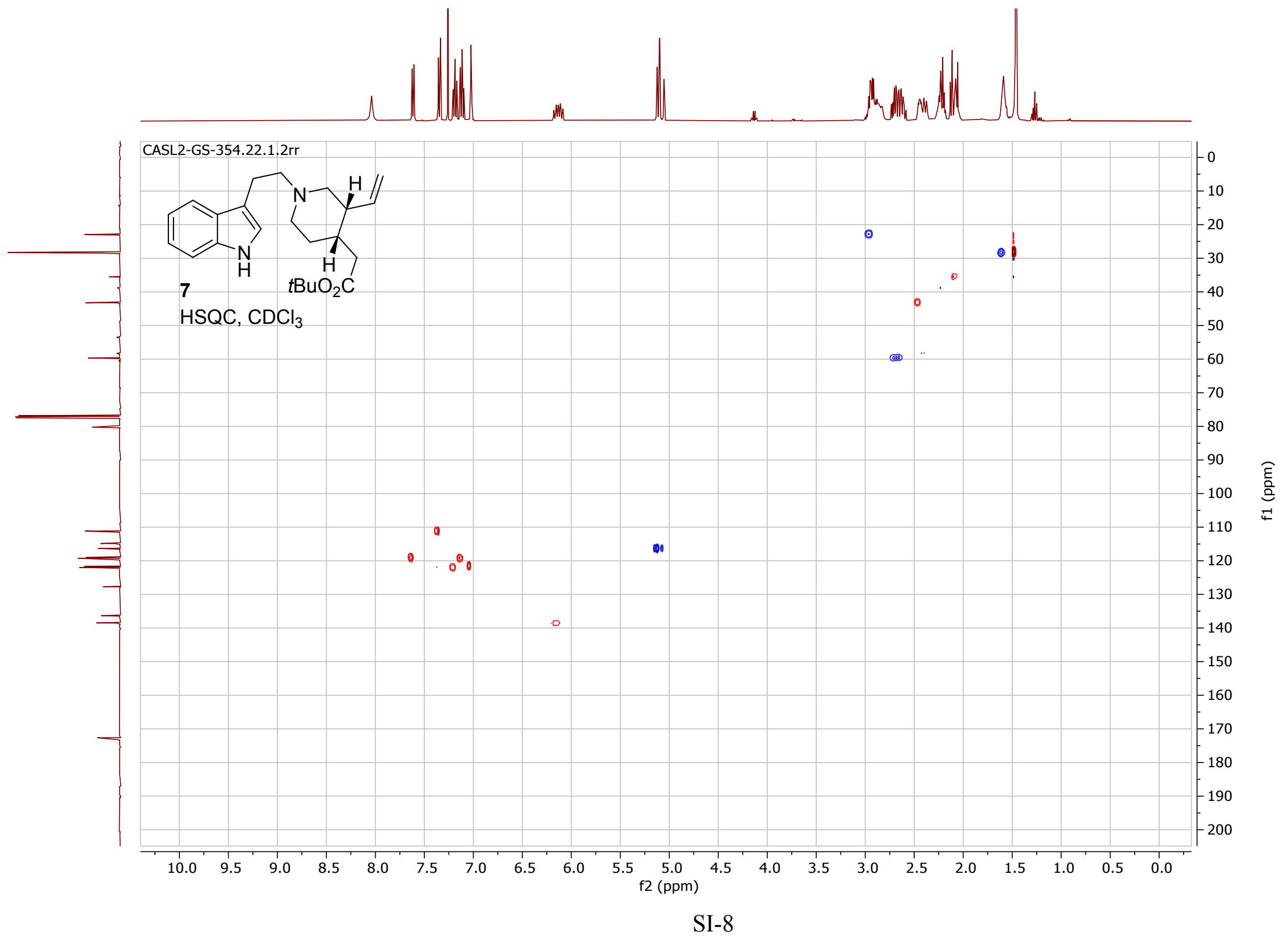


CASL2-GS-358-F1.10.1.1r

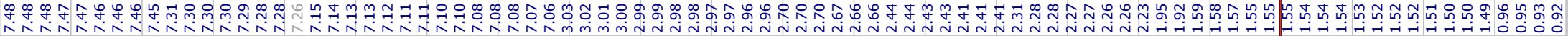

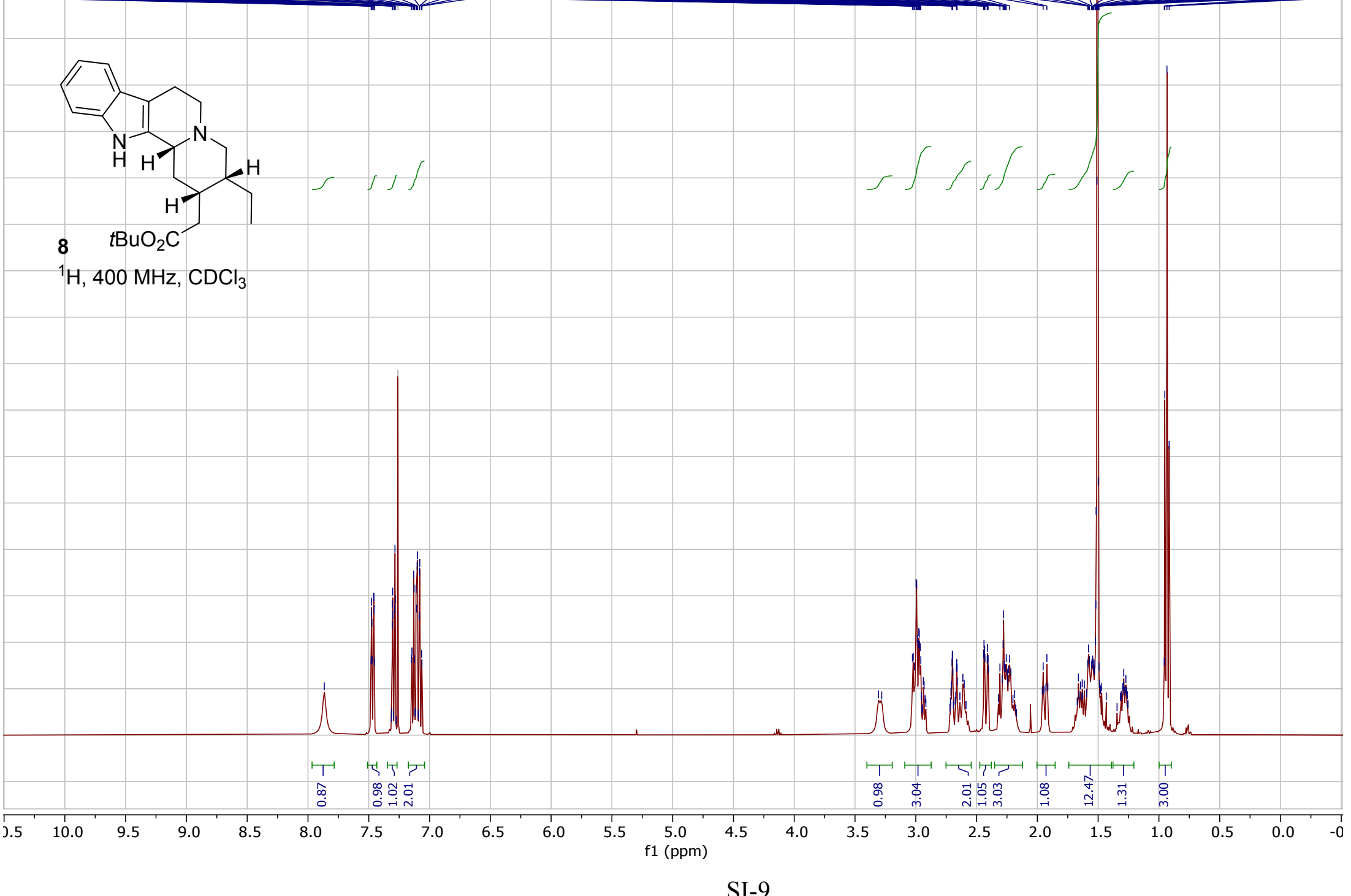




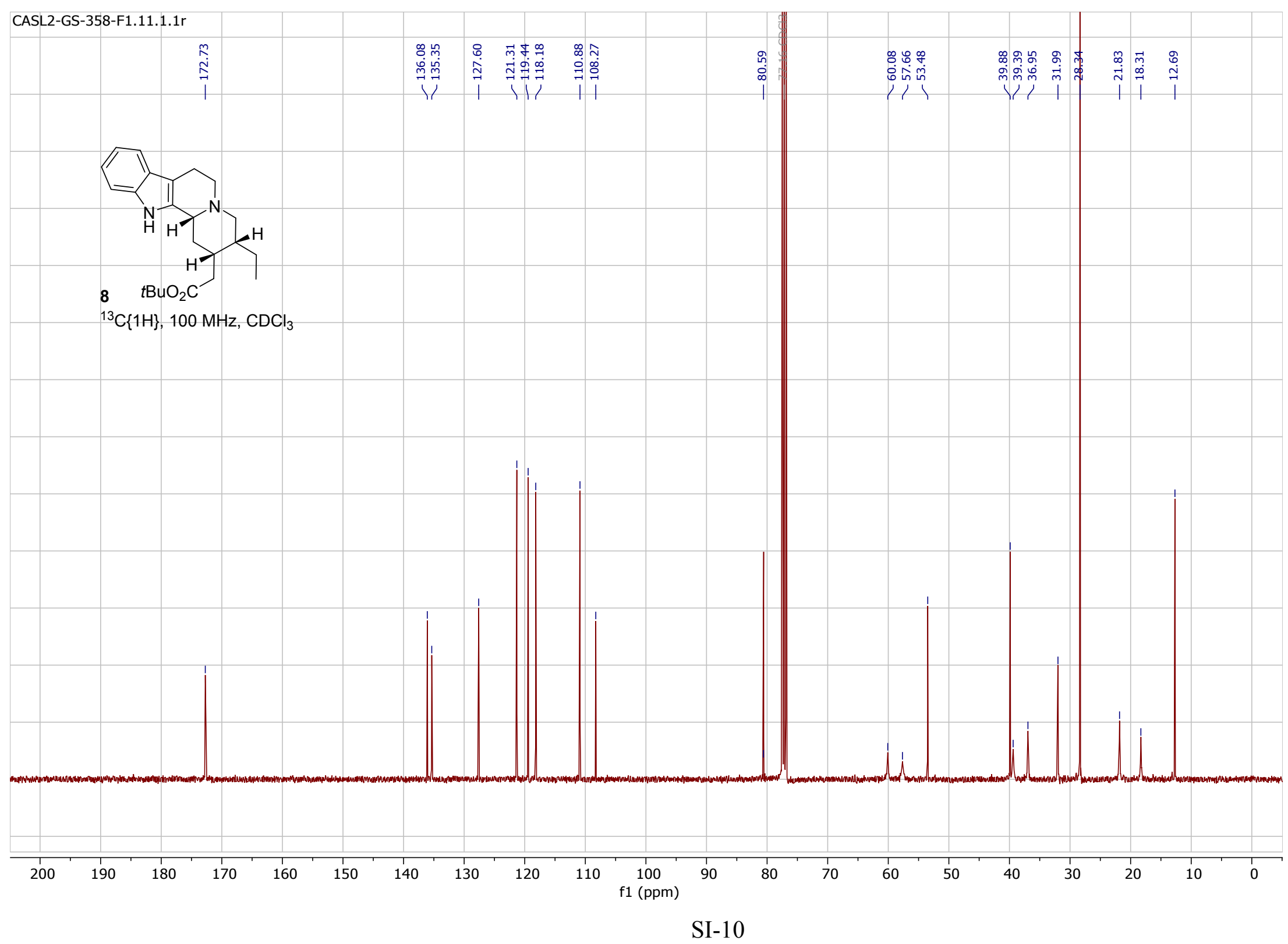




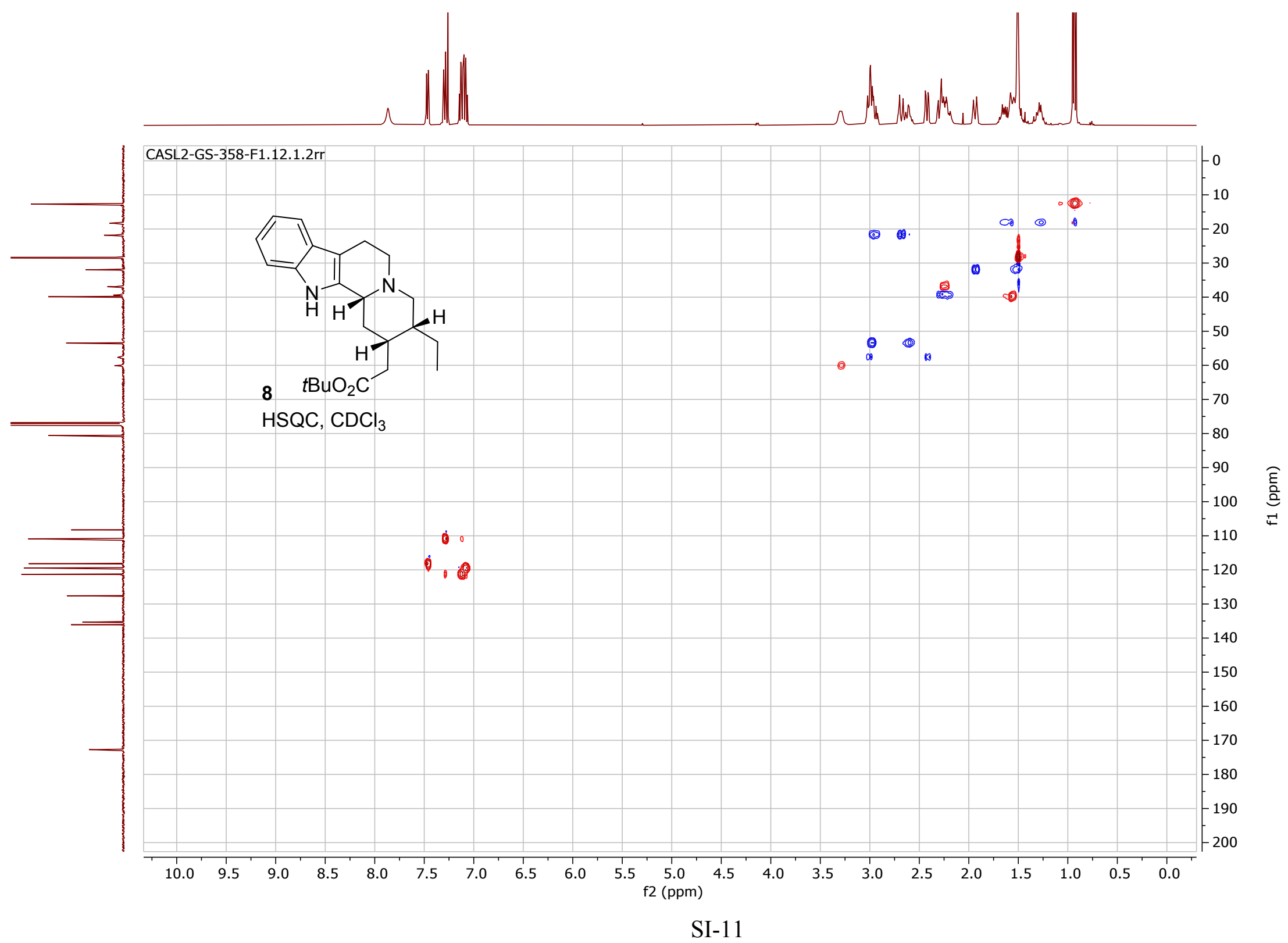




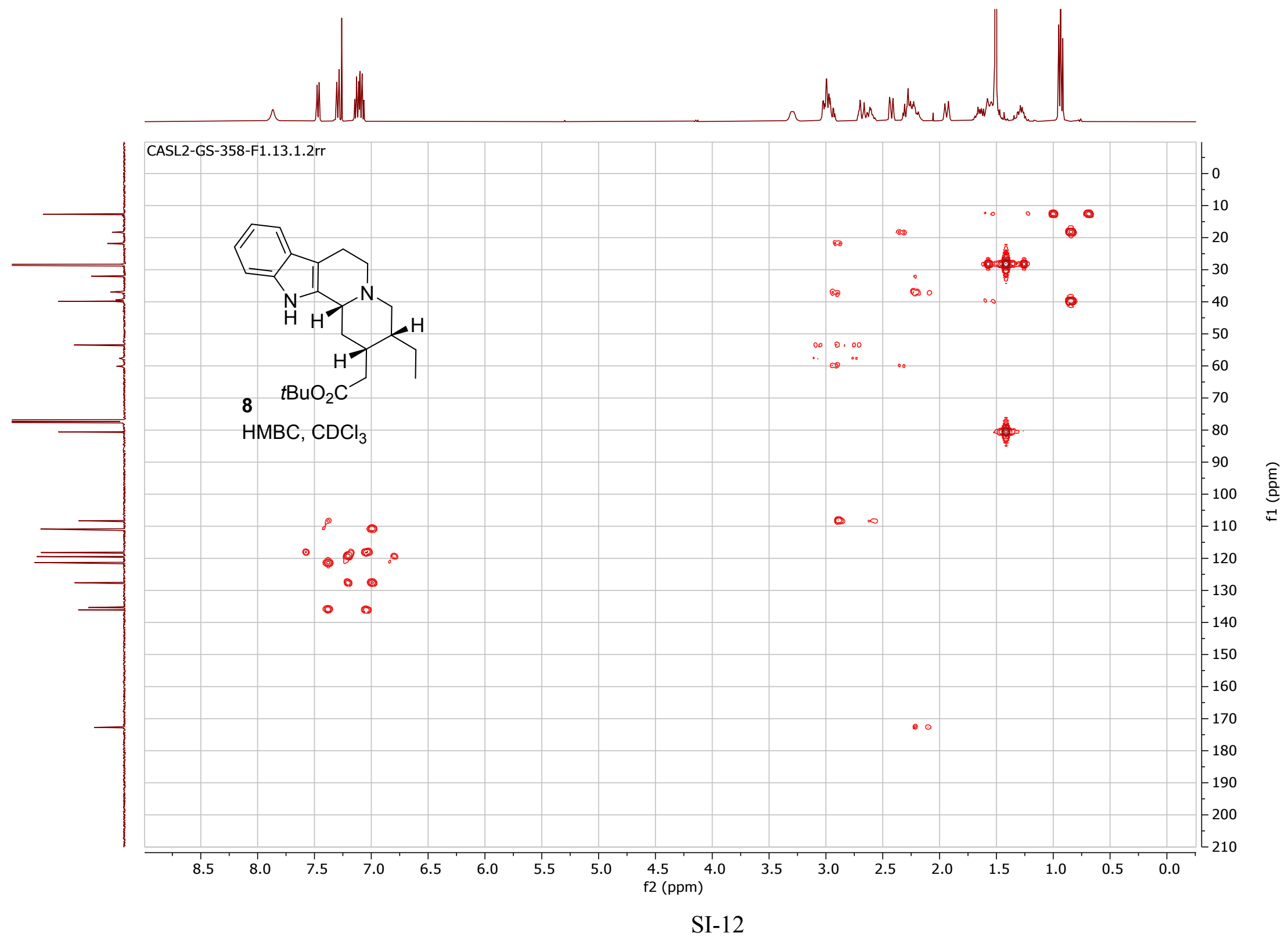




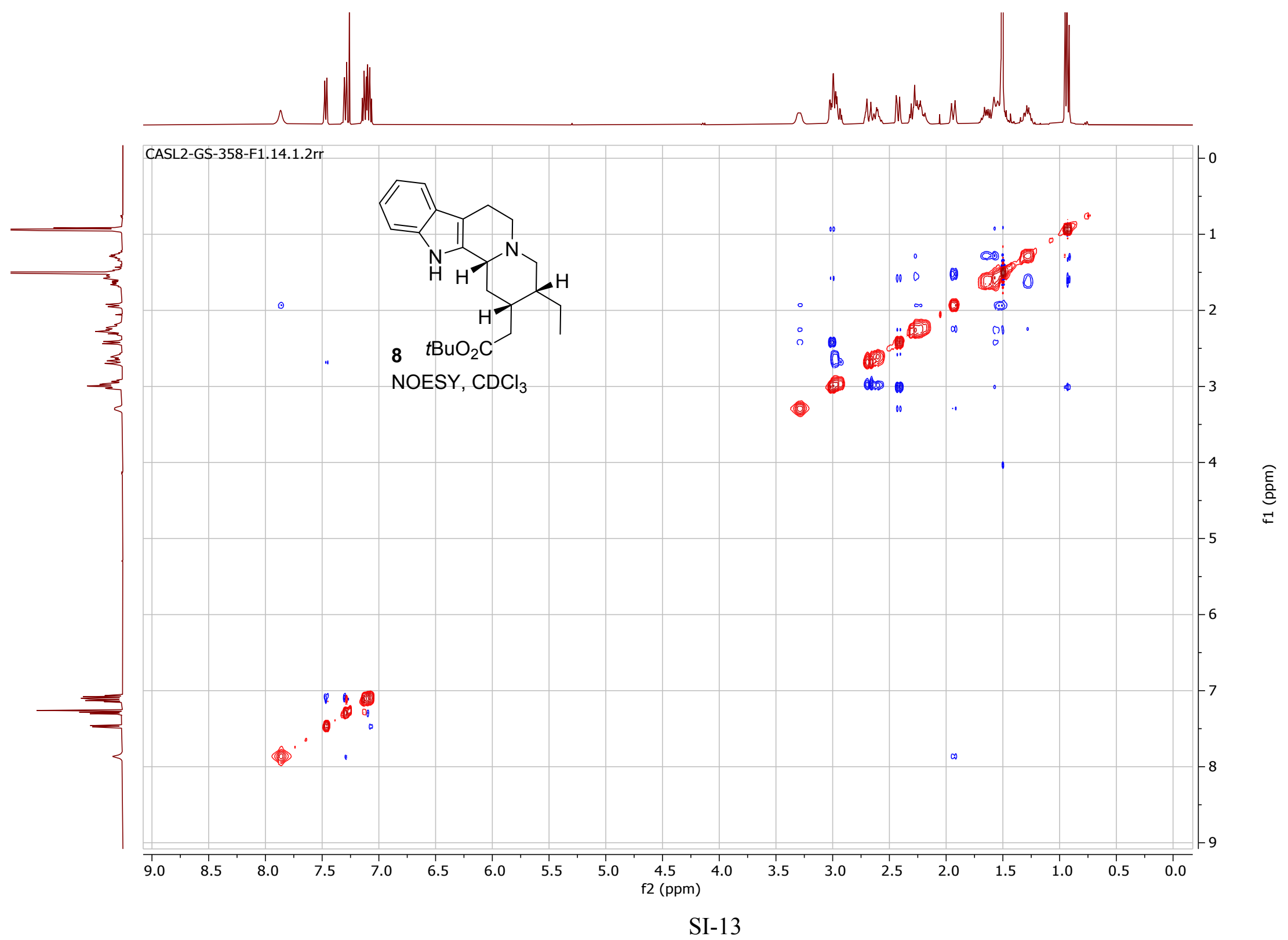


CASL2-GS-356-2.10.1.1r

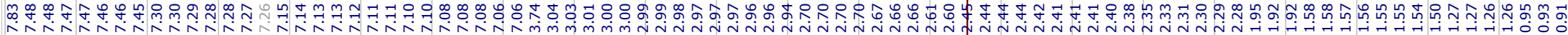

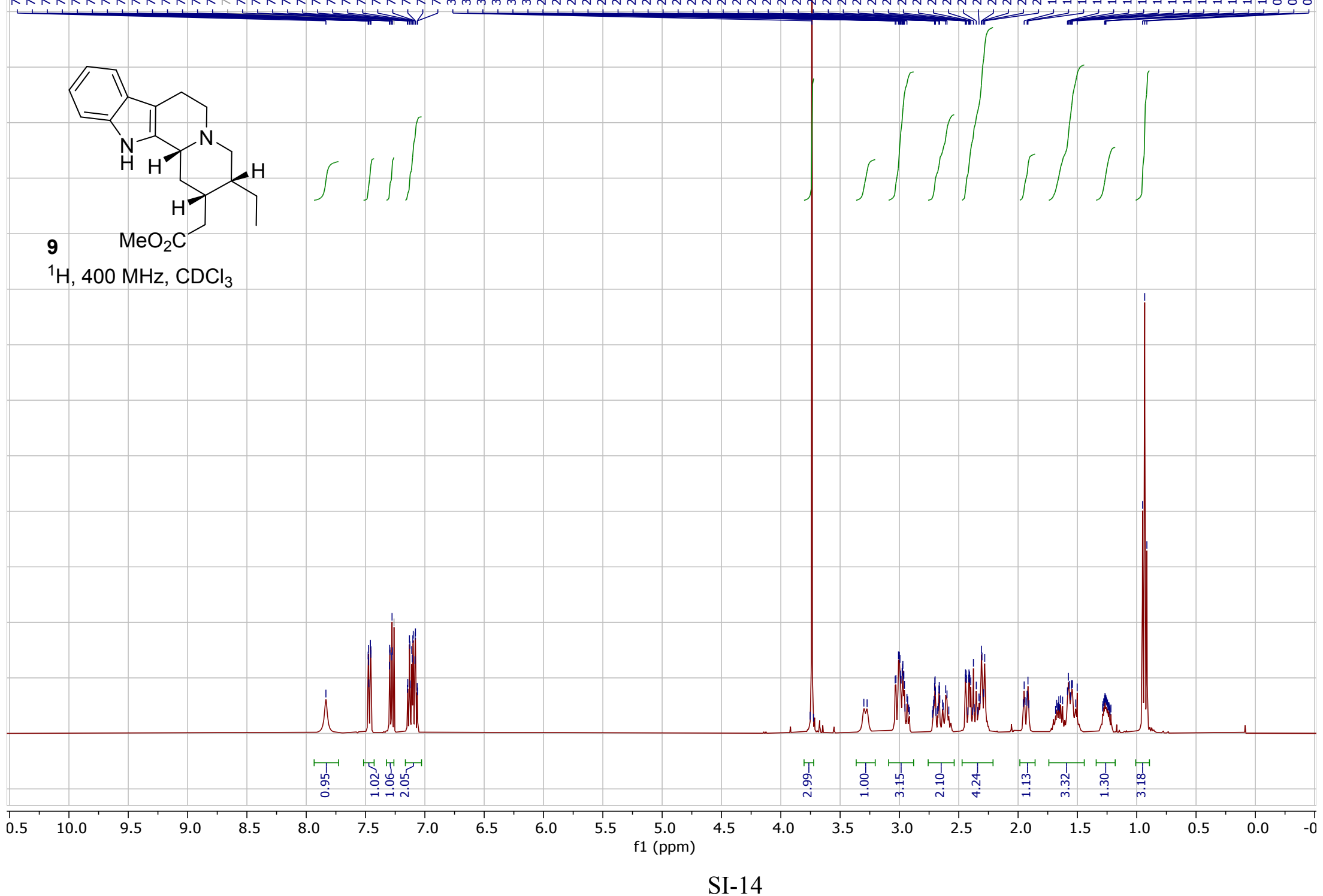




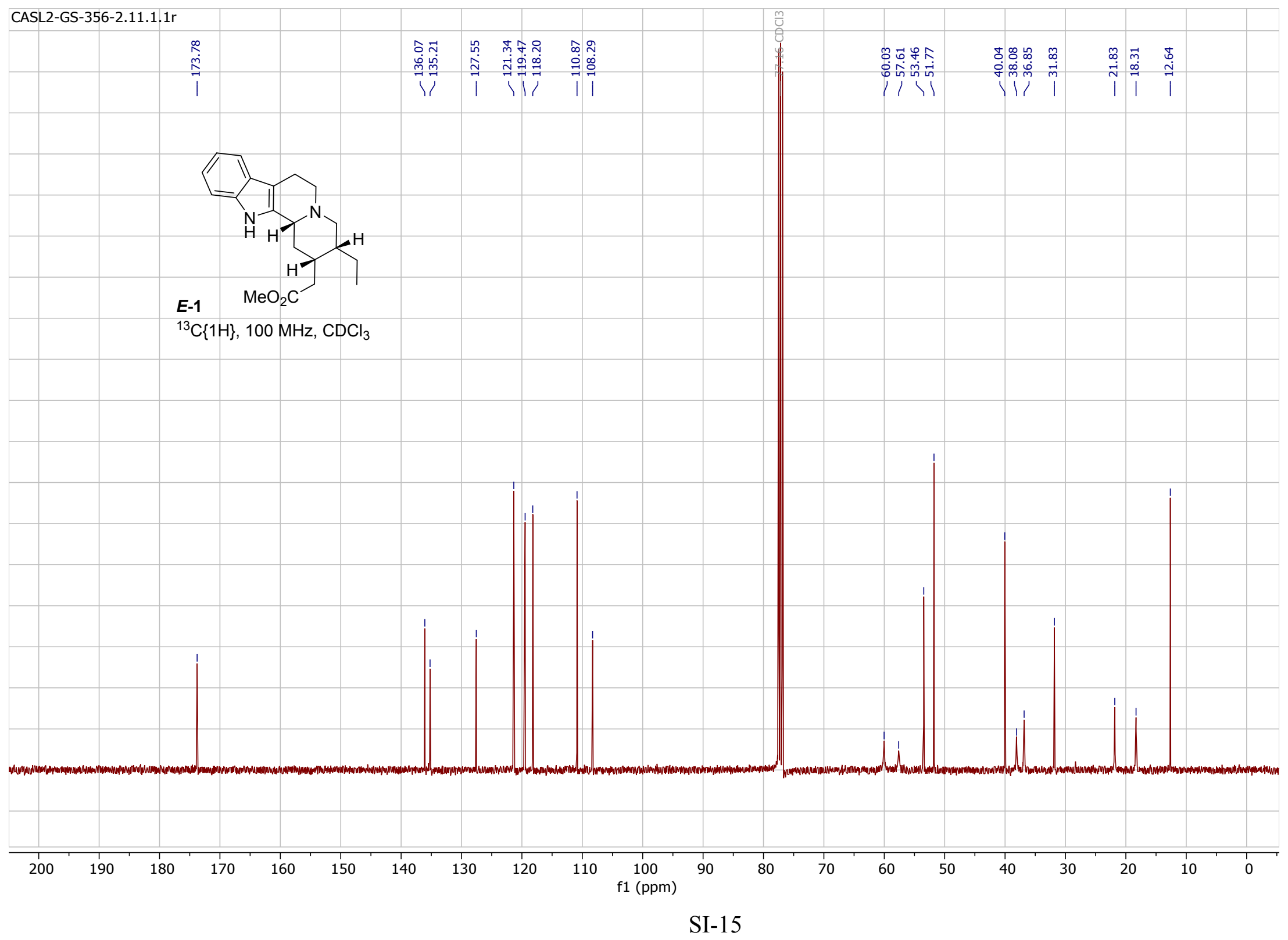




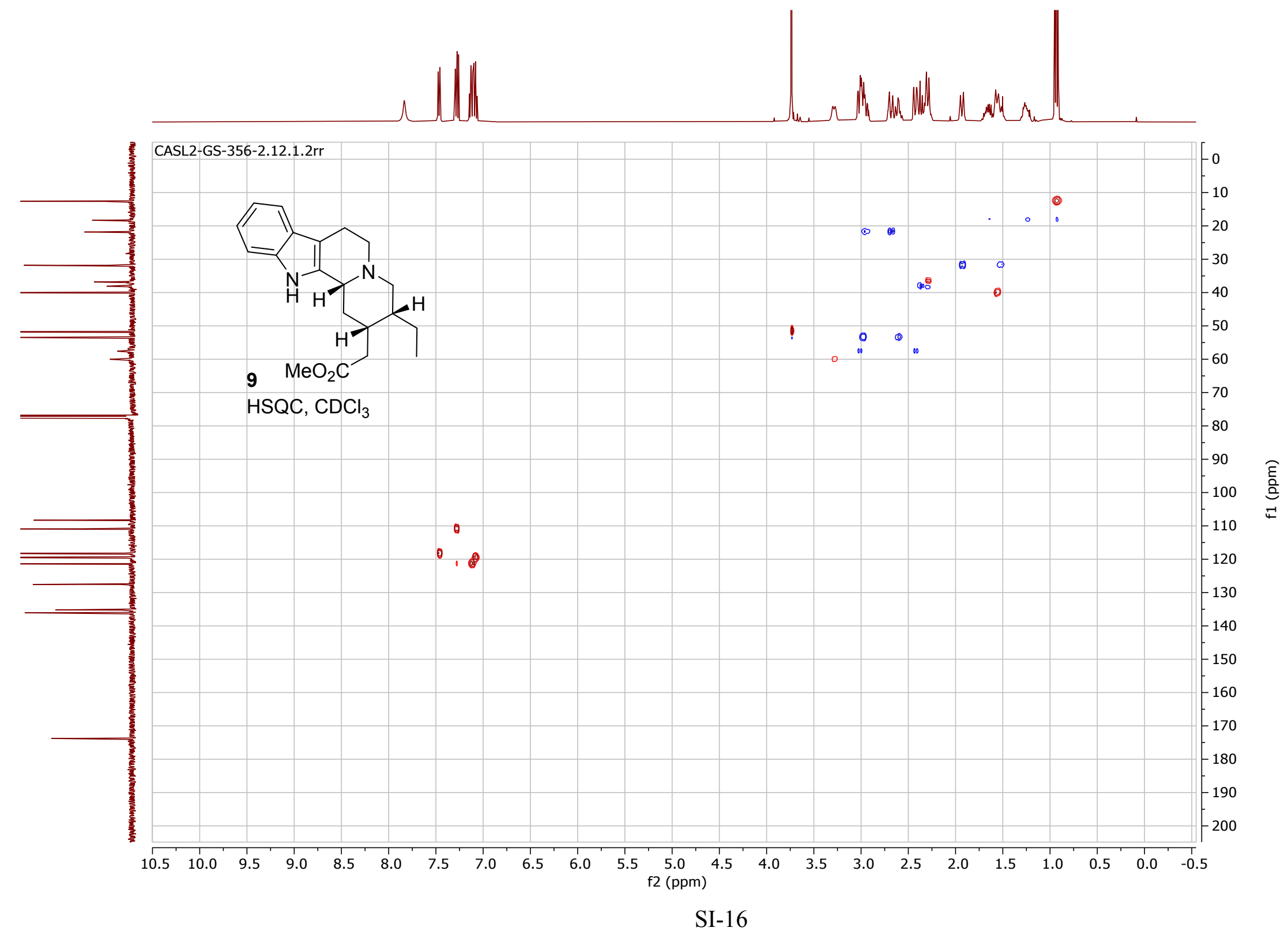


CASL2-GS-359F1.20.1.1r

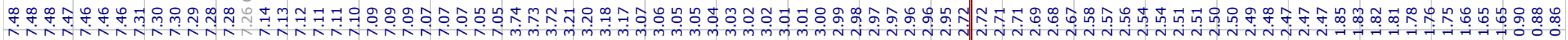

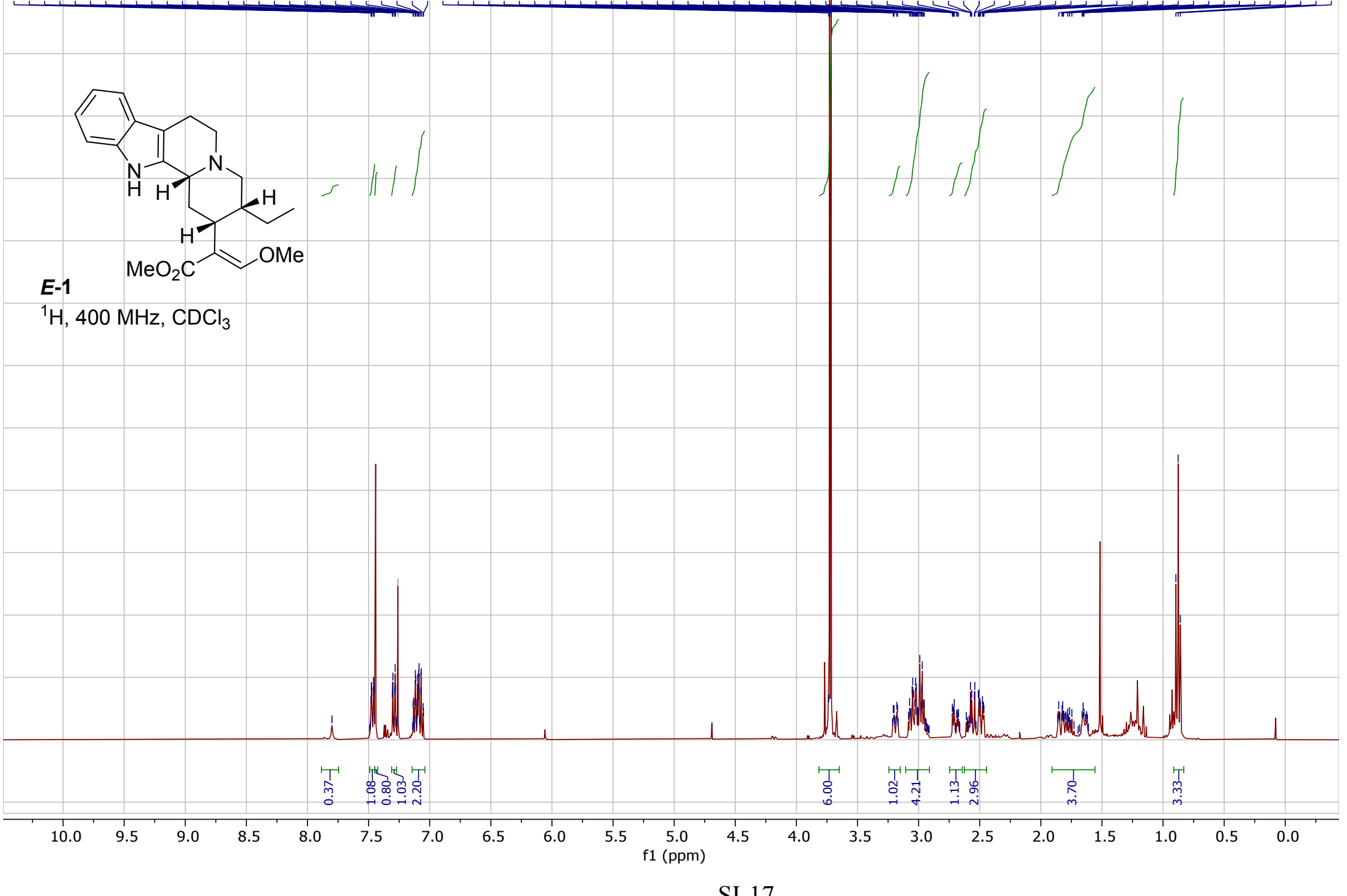

SI-17 


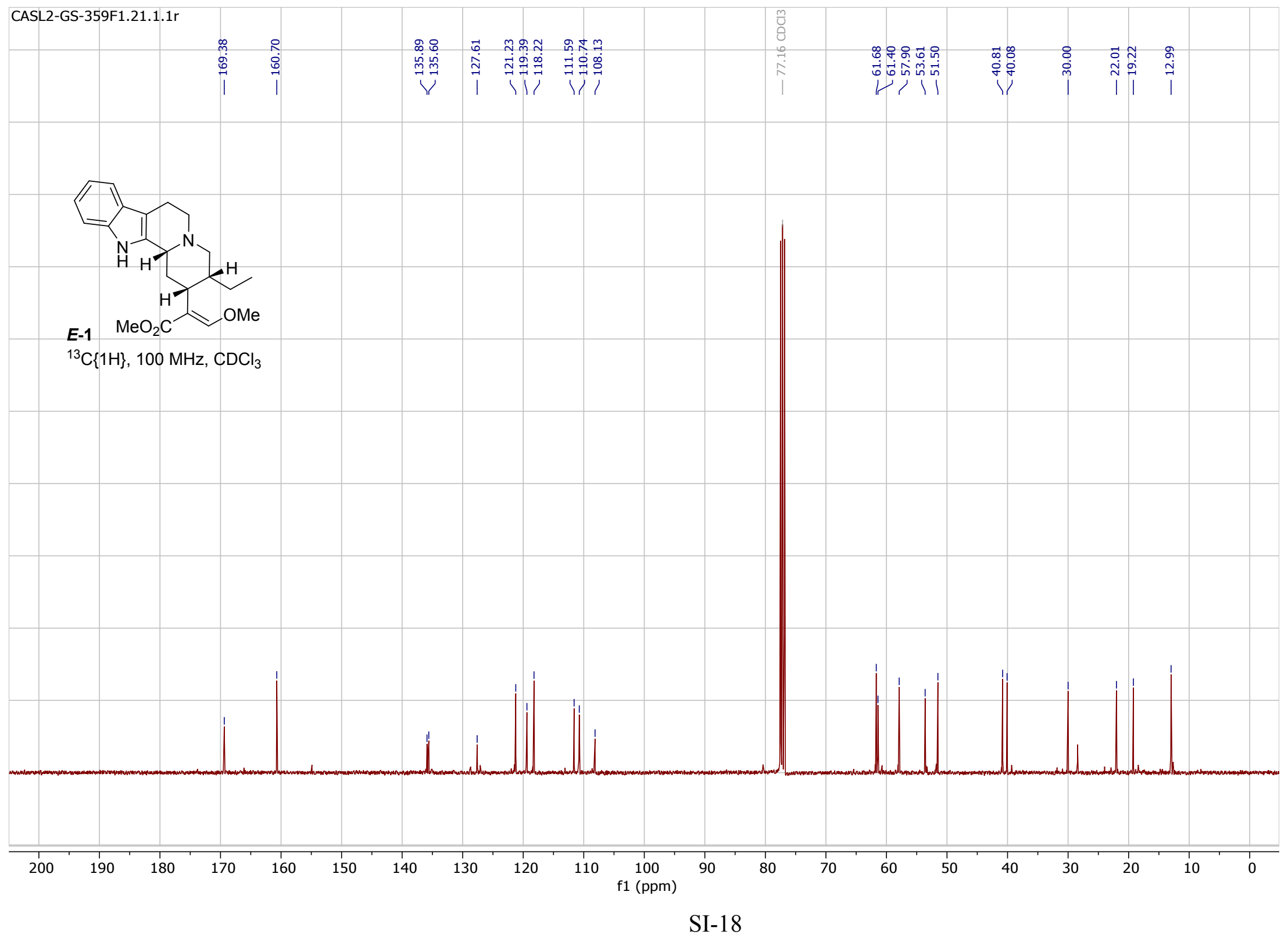


CASL2-GS-359F1.10.1.1r

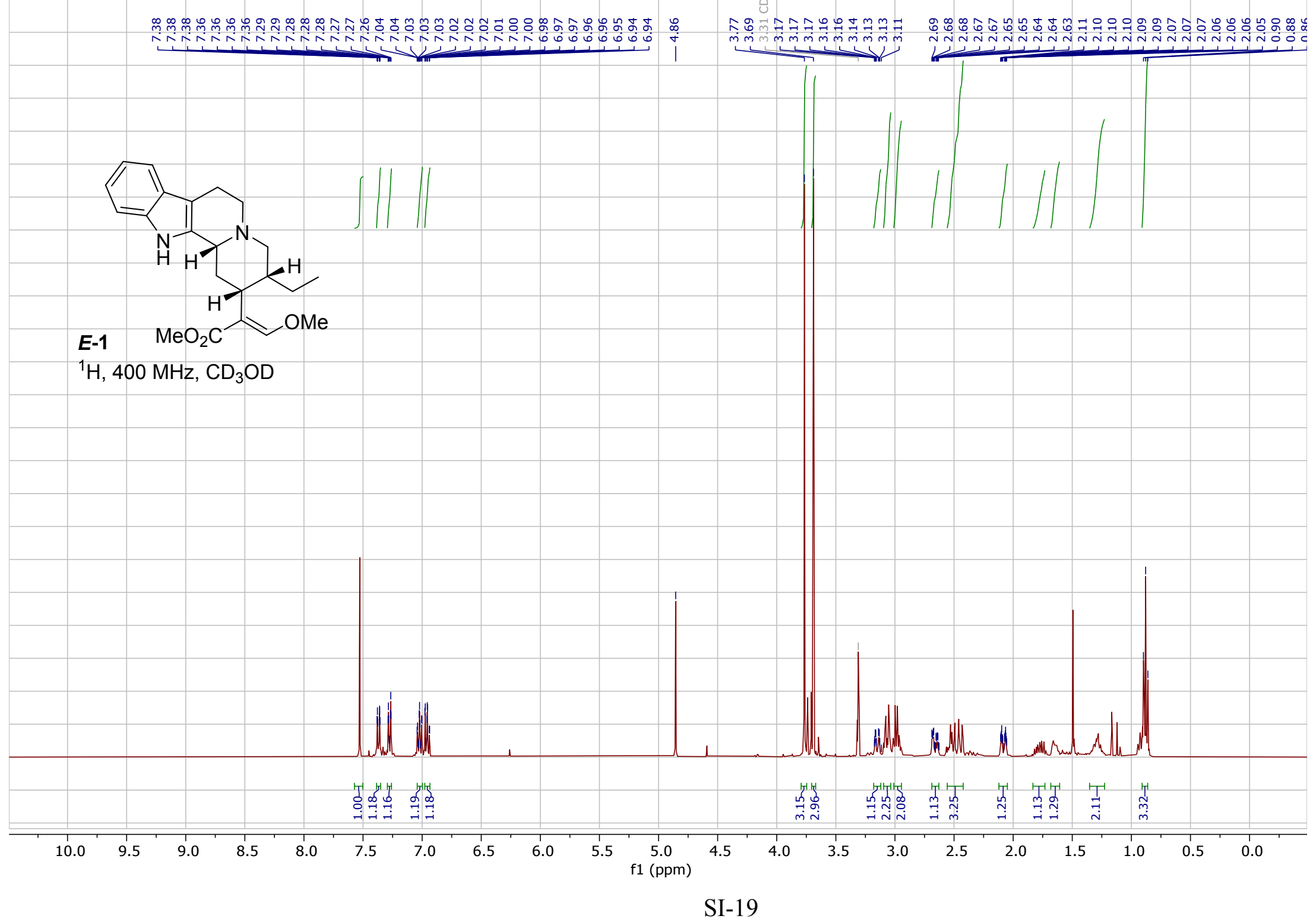




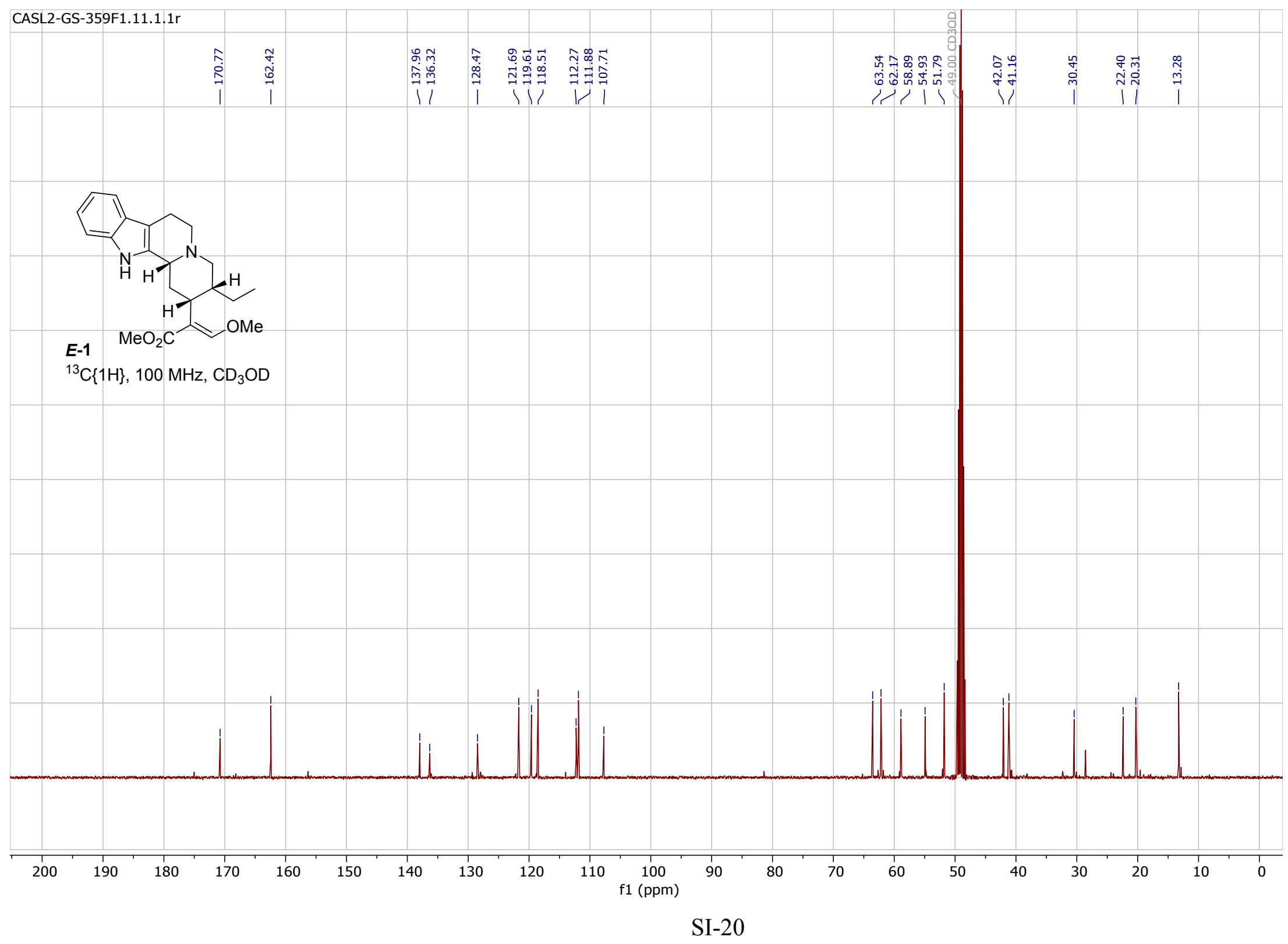




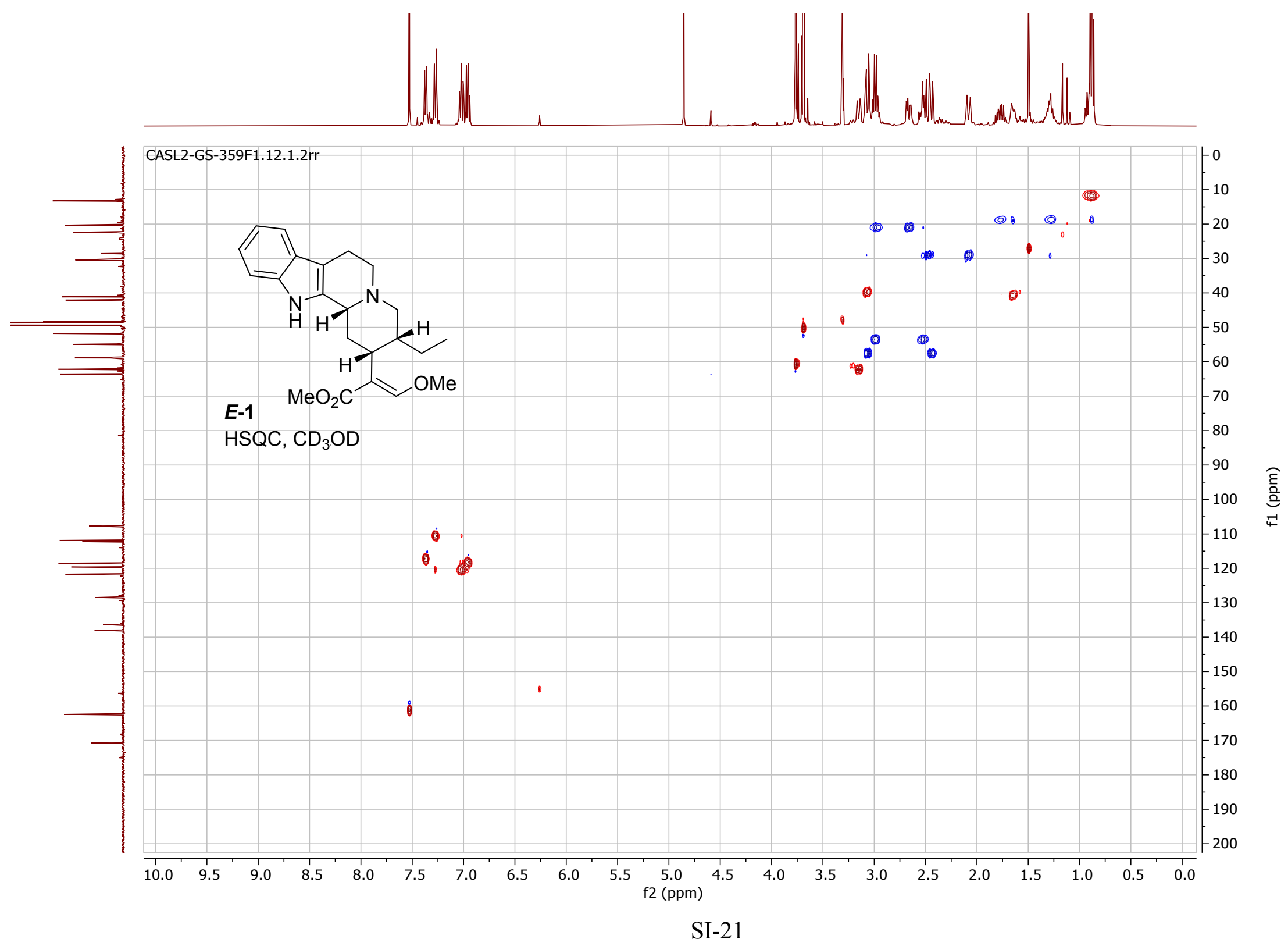




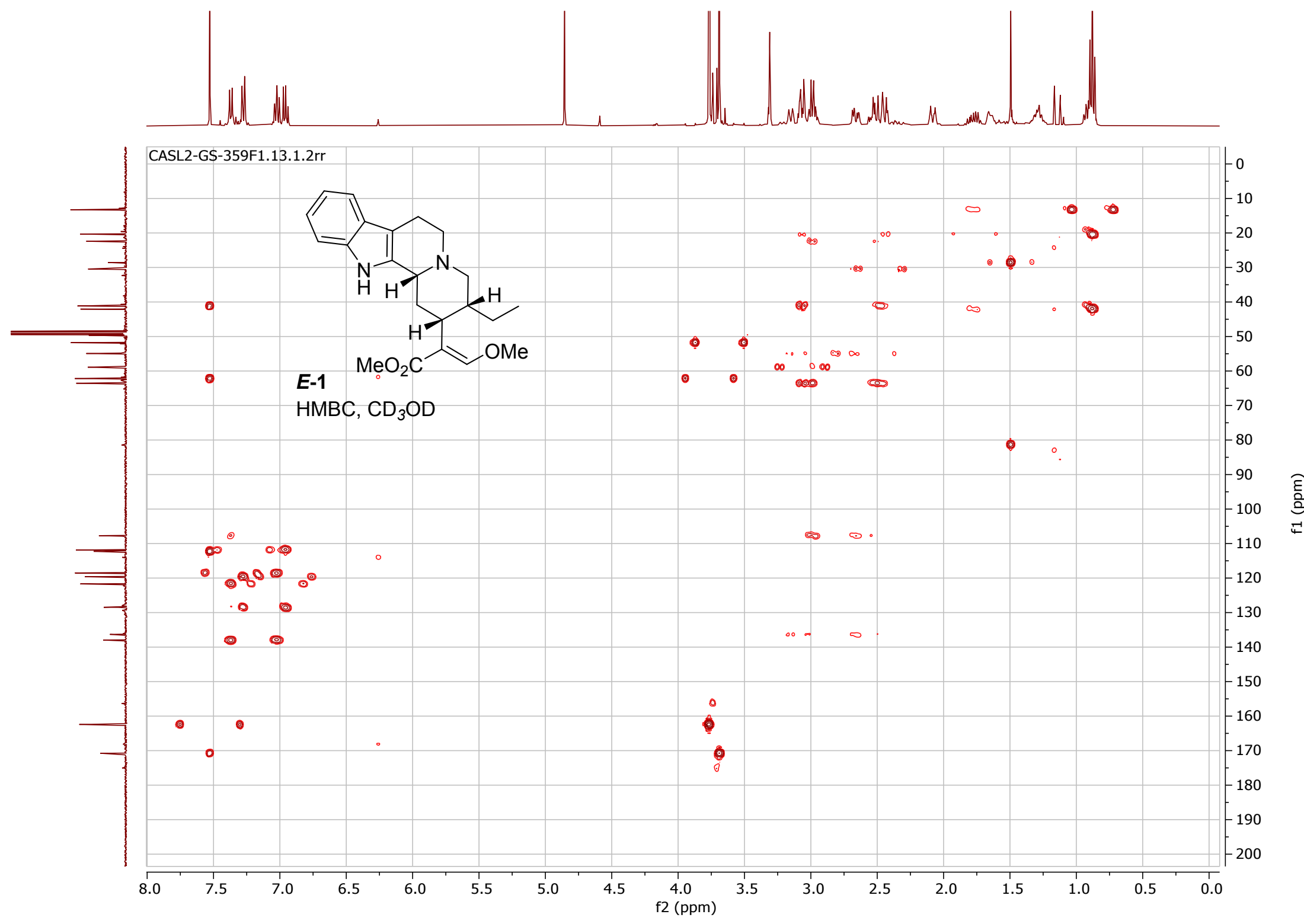

SI-22 


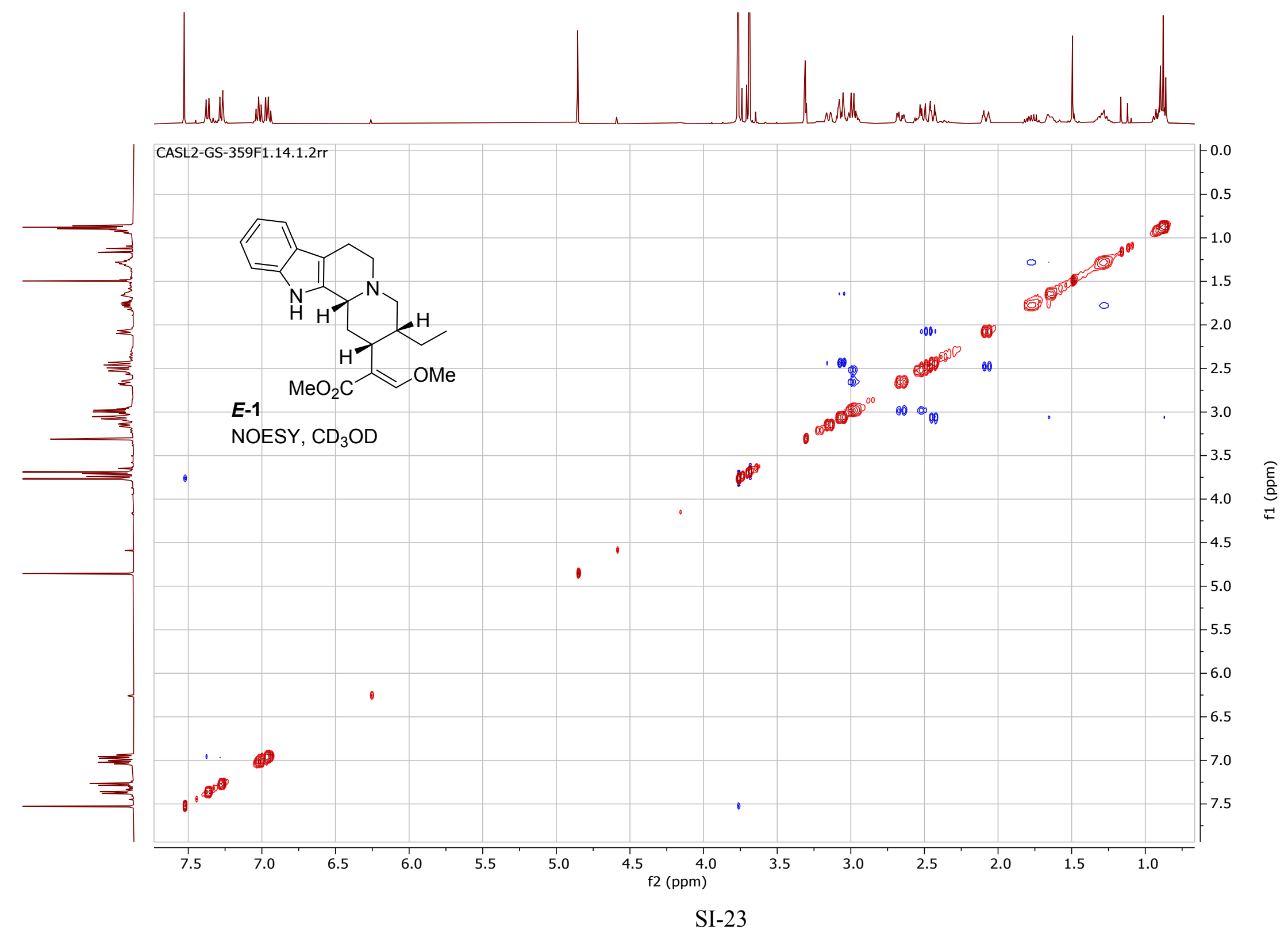




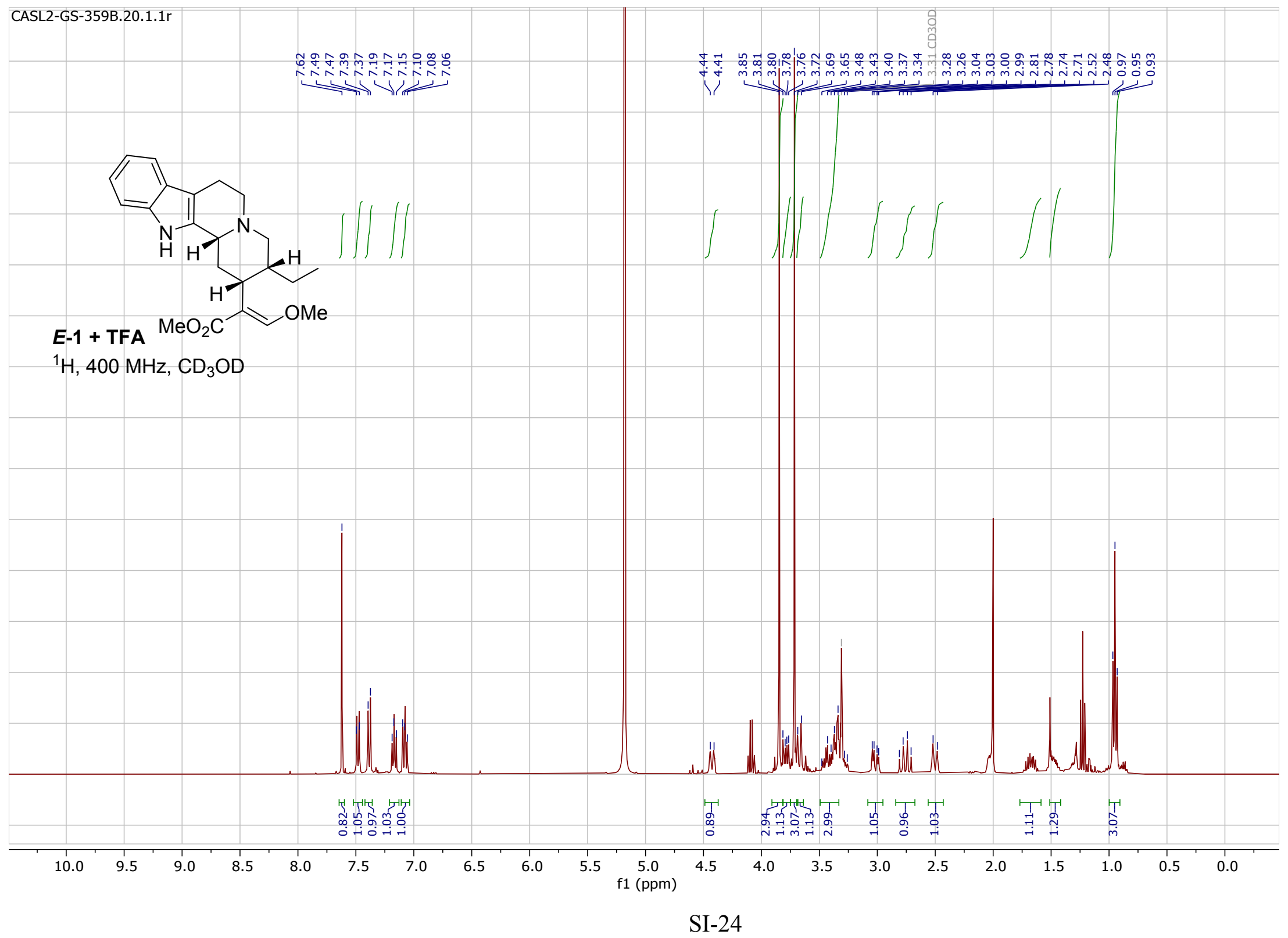




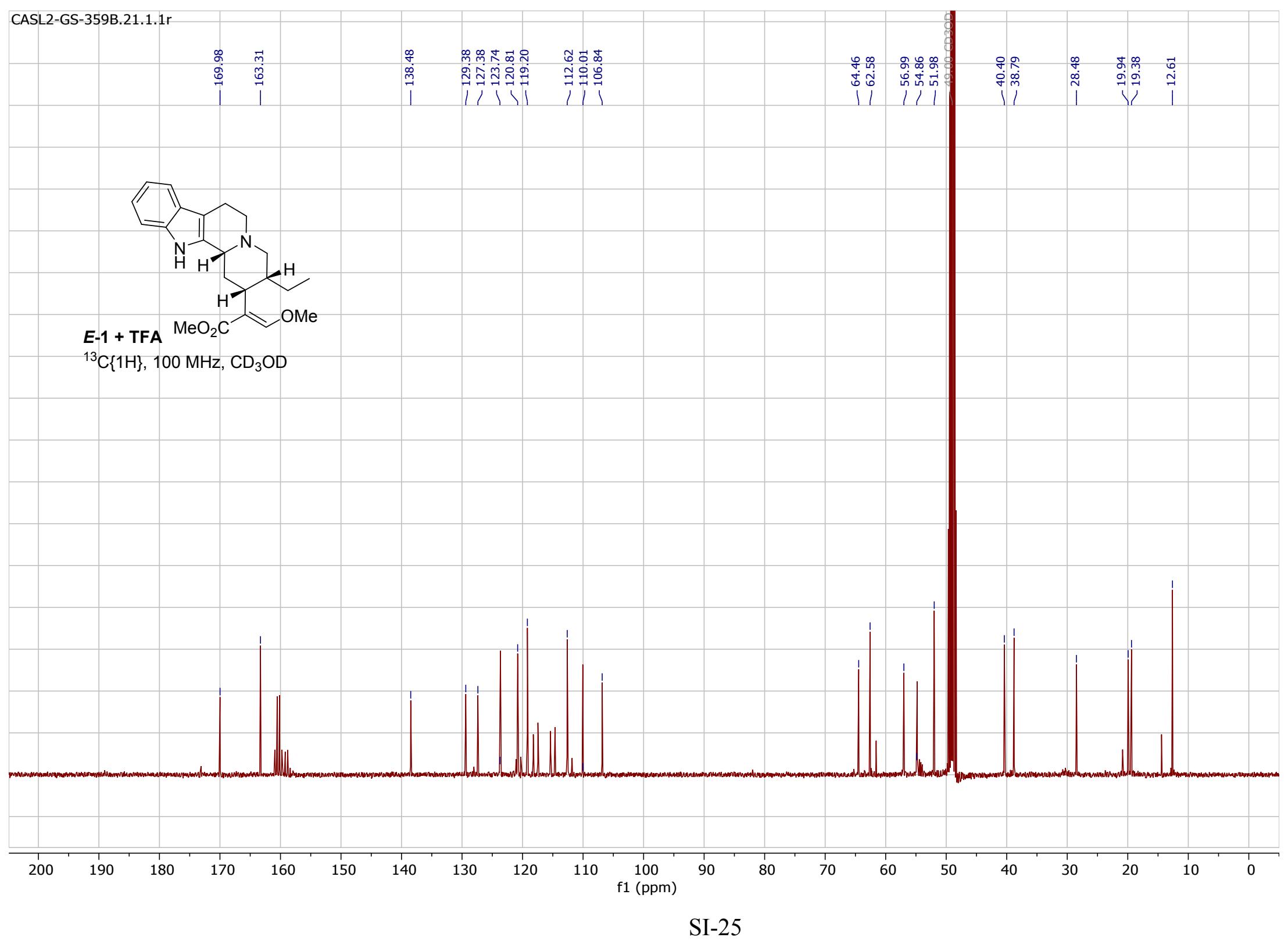




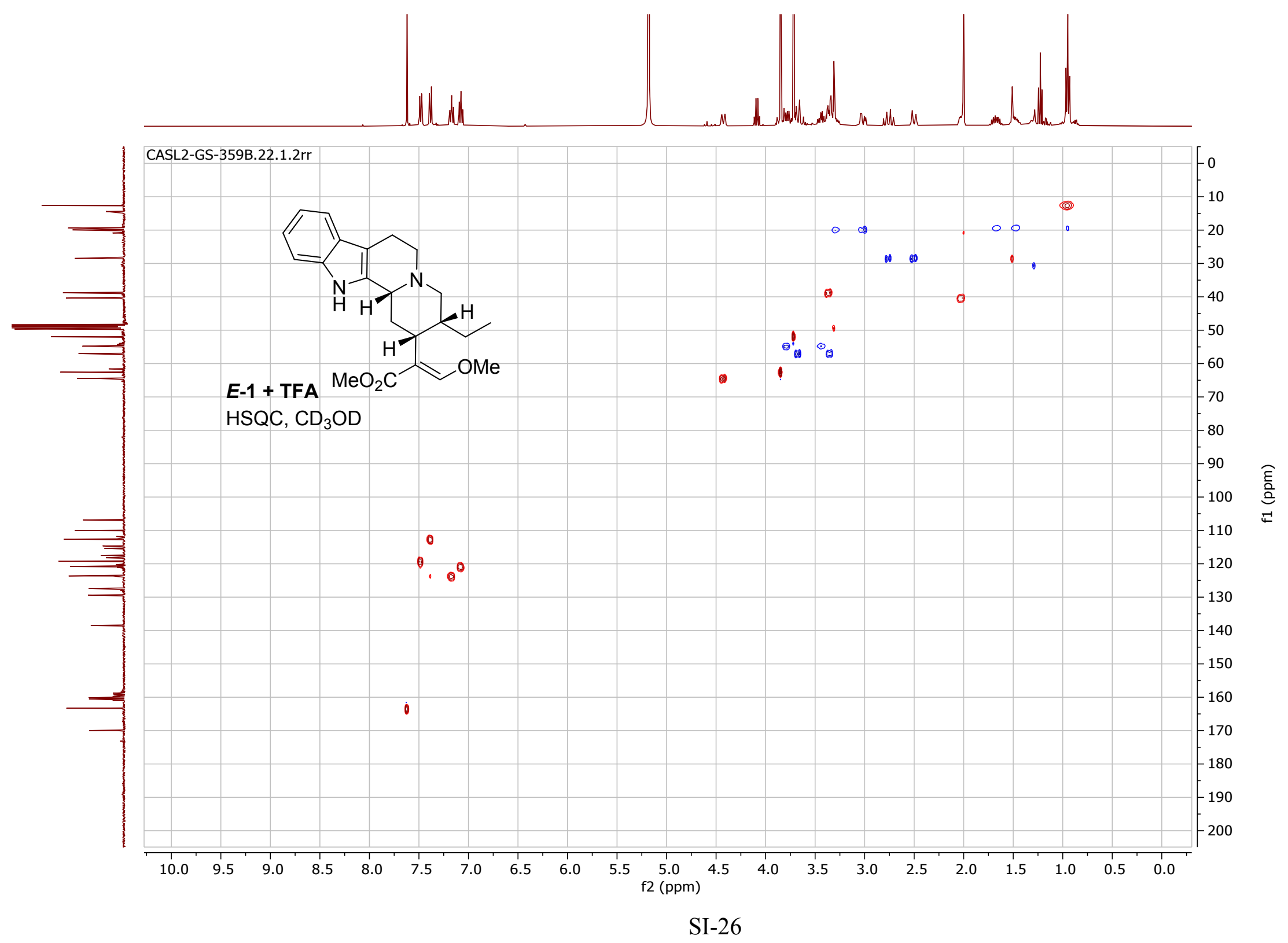




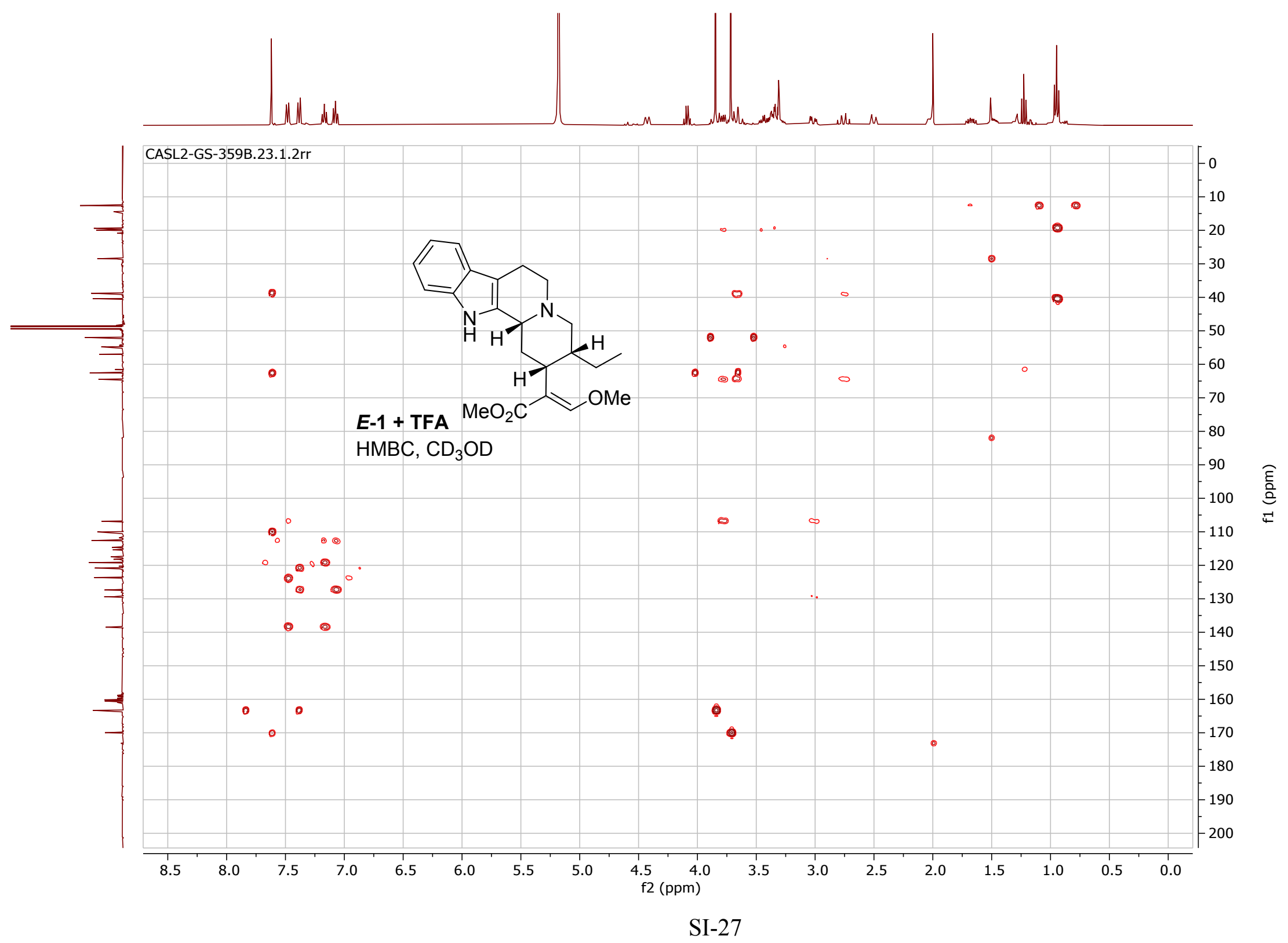


CASL2-GS-359-F2.11.1.1r

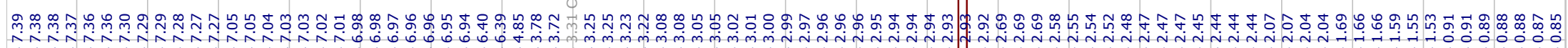

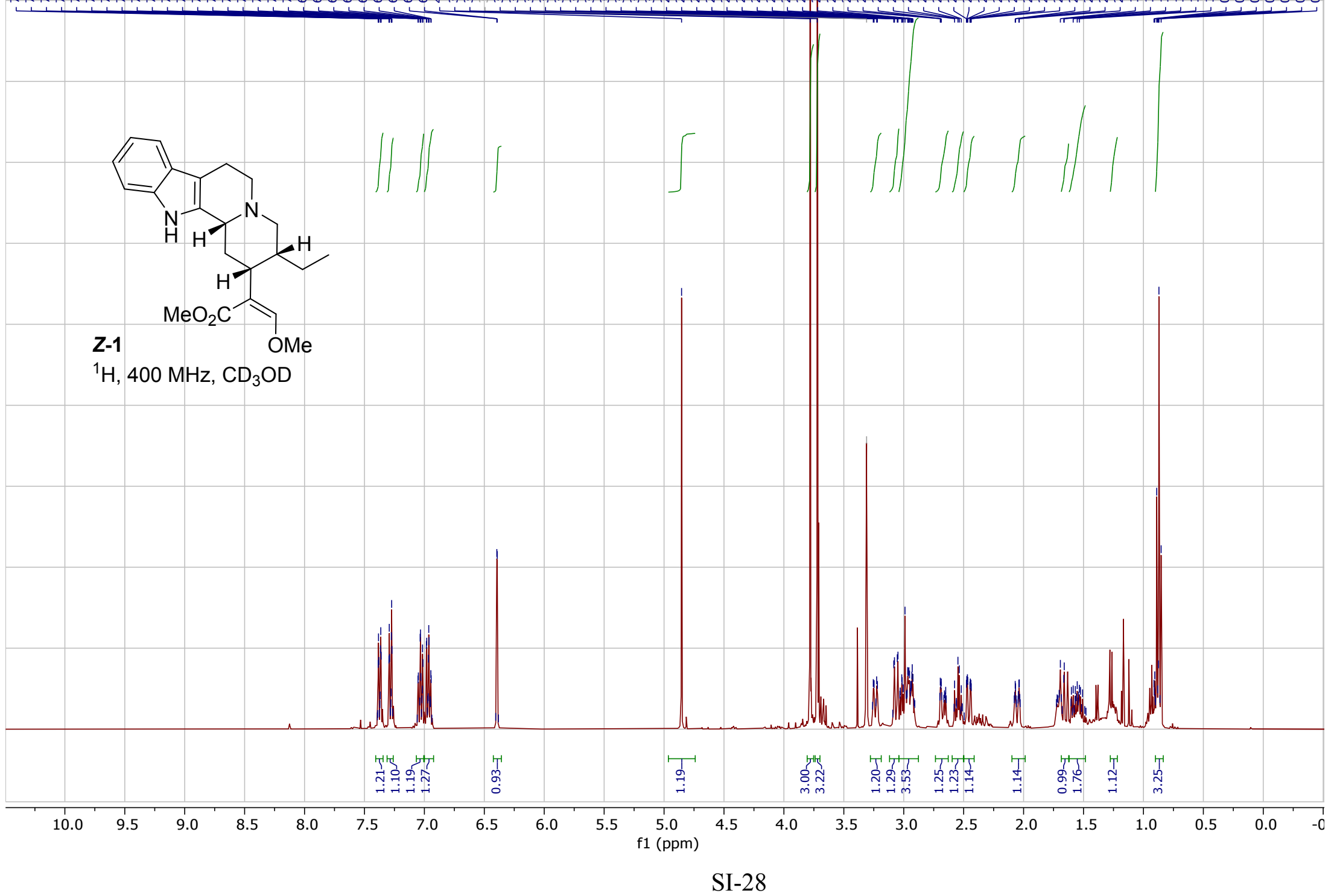




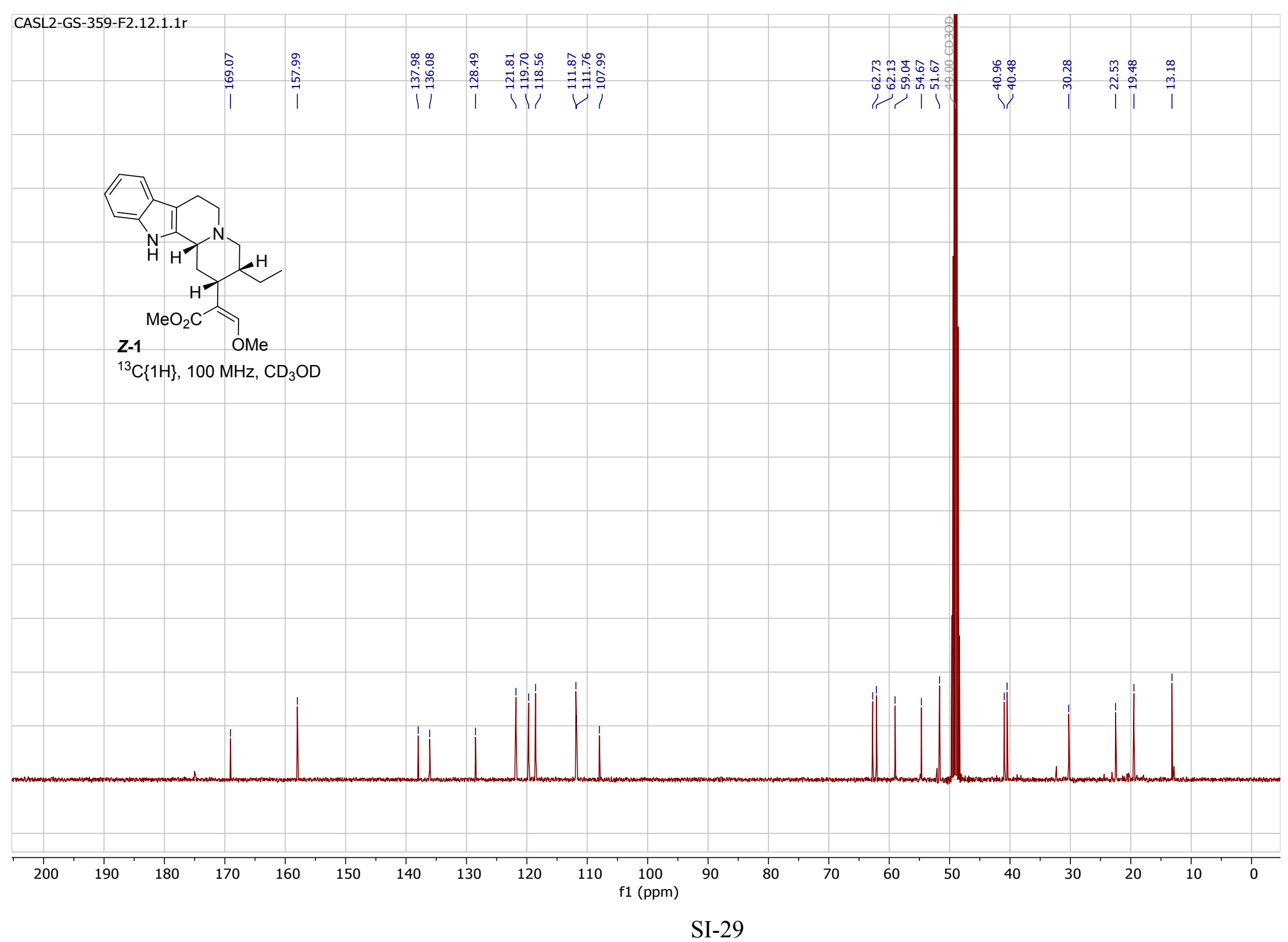




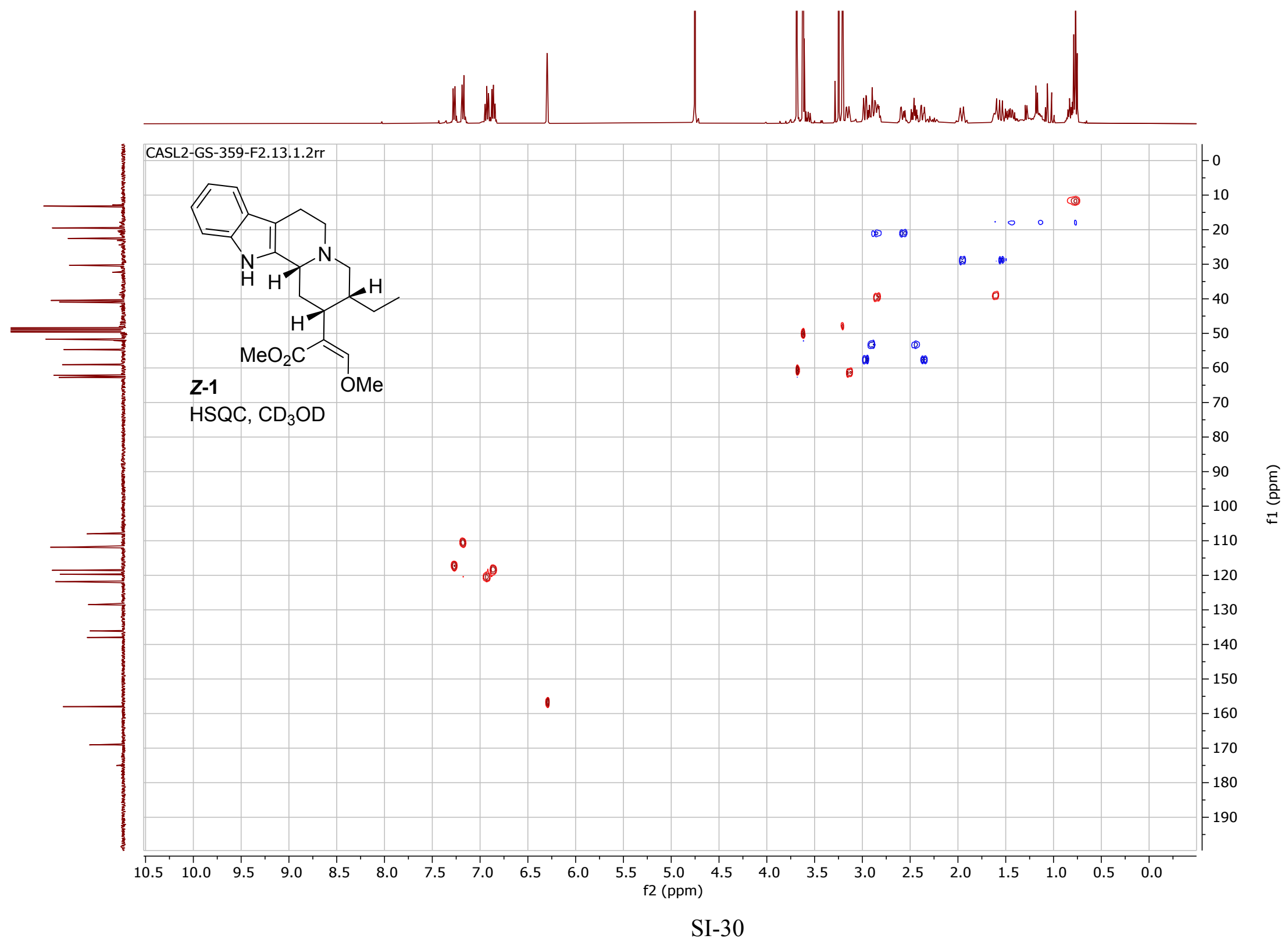




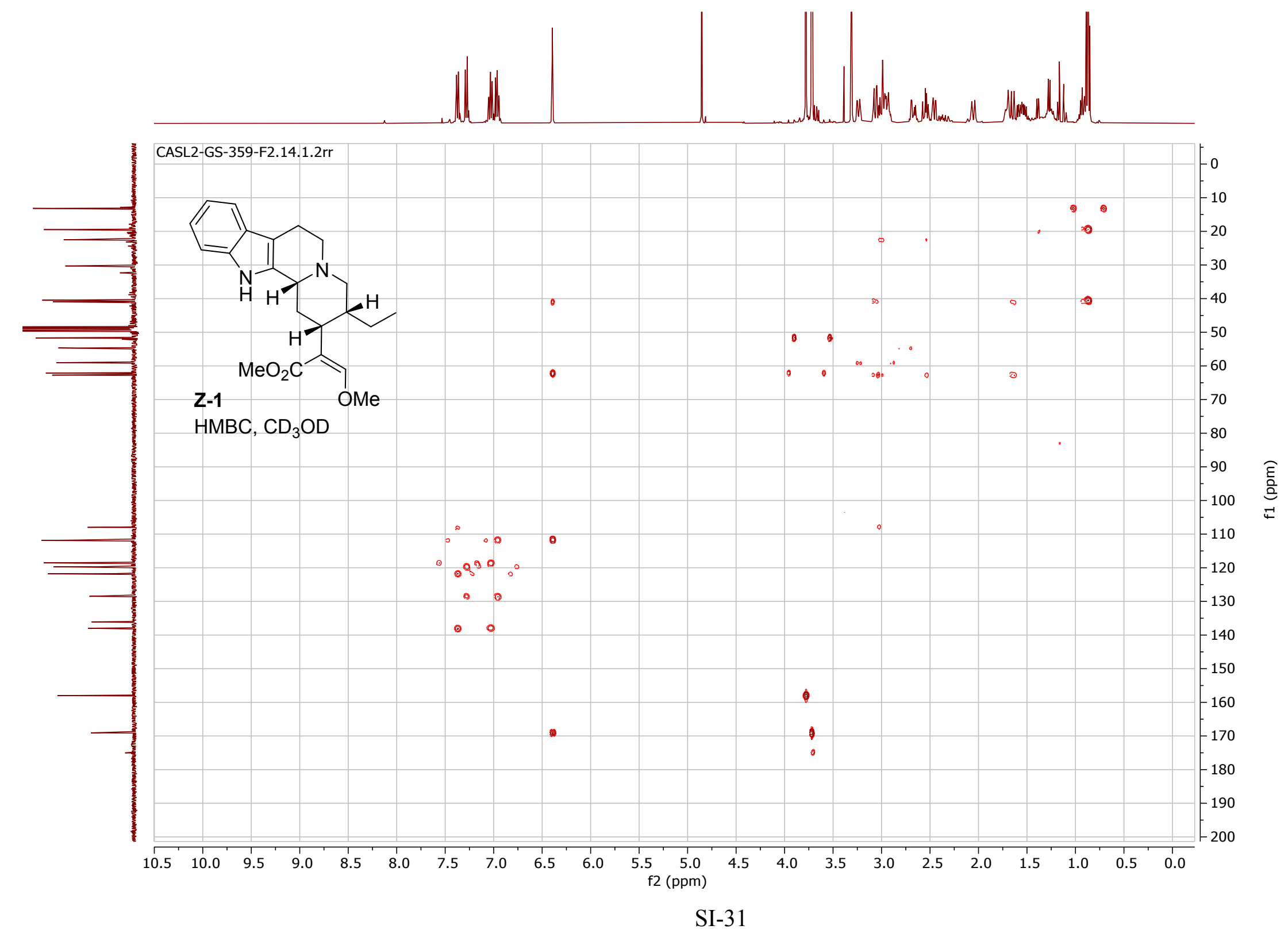




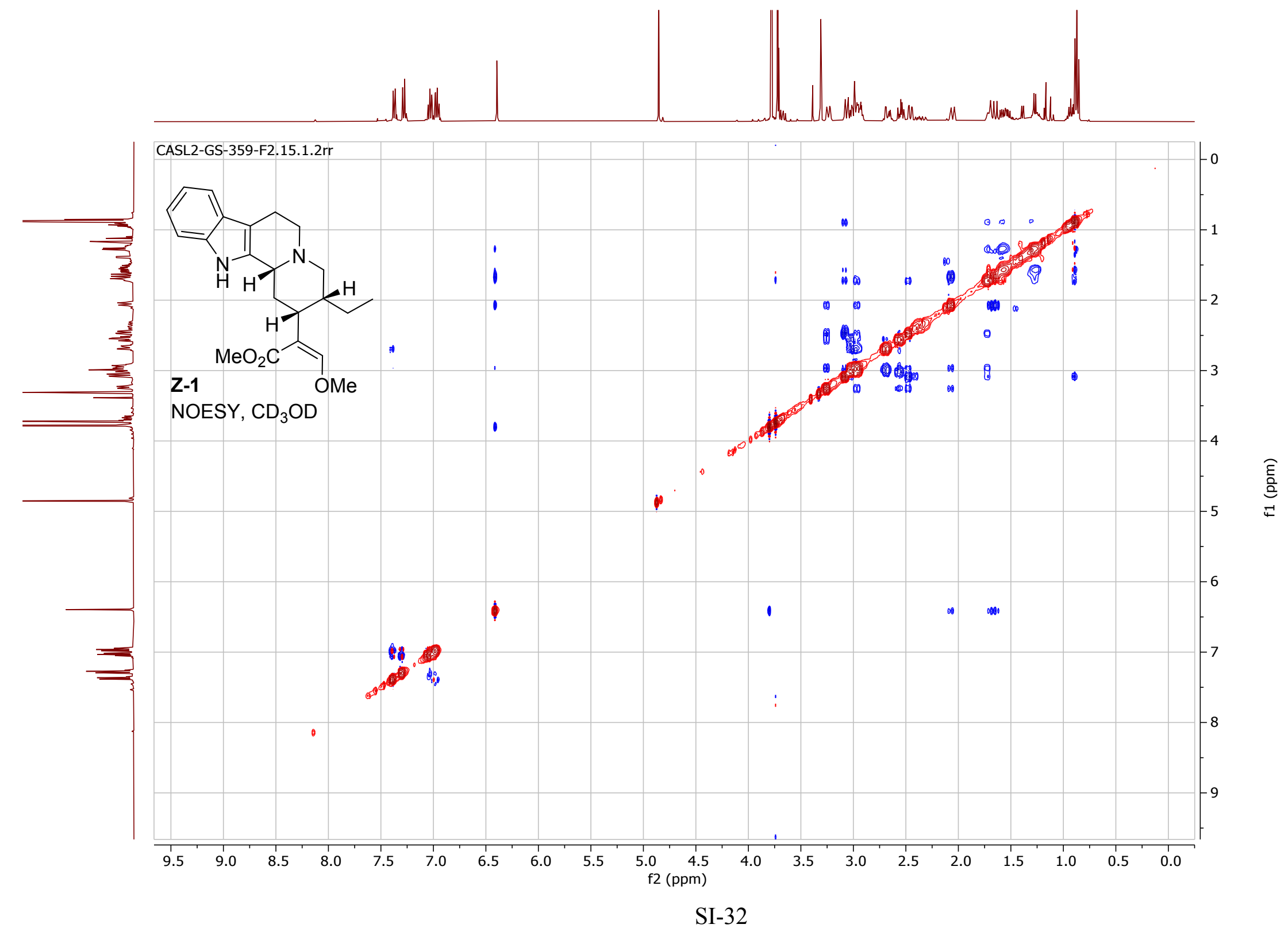

